\title{
Performance modelling of plasma microthruster nozzles in vacuum
}

Teck Seng Ho, Christine Charles, and Rod Boswell

Citation: Journal of Applied Physics 123, 173301 (2018); doi: 10.1063/1.5012765

View online: https://doi.org/10.1063/1.5012765

View Table of Contents: http://aip.scitation.org/toc/jap/123/17

Published by the American Institute of Physics

\section{Articles you may be interested in}

Electric force on plasma ions and the momentum of the ion-neutrals flow

Journal of Applied Physics 123, 173302 (2018); 10.1063/1.5007817

A polarization independent electromagnetically induced transparency-like metamaterial with large group delay and delay-bandwidth product

Journal of Applied Physics 123, 173101 (2018); 10.1063/1.5023684

Thrust performance, propellant ionization, and thruster erosion of an external discharge plasma thruster Journal of Applied Physics 123, 153302 (2018); 10.1063/1.5023829

Space micropropulsion systems for Cubesats and small satellites: From proximate targets to furthermost frontiers

Applied Physics Reviews 5, 011104 (2018); 10.1063/1.5007734

Rate equation analysis and non-Hermiticity in coupled semiconductor laser arrays

Journal of Applied Physics 123, 173102 (2018); 10.1063/1.5022044

Electroluminescent refrigeration by ultra-efficient GaAs light-emitting diodes

Journal of Applied Physics 123, 173104 (2018); 10.1063/1.5019764

AIP/ Iopplied of Physics SPECIAL TOPICS

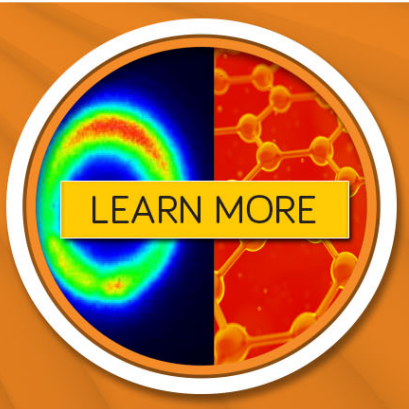




\title{
Performance modelling of plasma microthruster nozzles in vacuum
}

\author{
Teck Seng Ho, Christine Charles, and Rod Boswell \\ Space Plasma, Power and Propulsion Laboratory, Research School of Physics and Engineering, \\ The Australian National University, Canberra, ACT 2601, Australia
}

(Received 7 November 2017; accepted 5 April 2018; published online 1 May 2018)

\begin{abstract}
Computational fluid dynamics and plasma simulations of three geometrical variations of the Pocket Rocket radiofrequency plasma electrothermal microthruster are conducted, comparing pulsed plasma to steady state cold gas operation. While numerical limitations prevent plasma modelling in a vacuum environment, results may be obtained by extrapolating from plasma simulations performed in a pressurised environment, using the performance delta from cold gas simulations performed in both environments. Slip regime boundary layer effects are significant at these operating conditions. The present investigation targets a power budget of $\sim 10 \mathrm{~W}$ for applications on CubeSats. During plasma operation, the thrust force increases by $\sim 30 \%$ with a power efficiency of $\sim 30 \mu \mathrm{NW}^{-1}$. These performance metrics represent instantaneous or pulsed operation and will increase over time as the discharge chamber attains thermal equilibrium with the heated propellant. Additionally, the sculpted nozzle geometry achieves plasma confinement facilitated by the formation of a plasma sheath at the nozzle throat, and fast recombination ensures a neutral exhaust plume that avoids the contamination of solar panels and interference with externally mounted instruments. Published by AIP Publishing. https://doi.org/10.1063/1.5012765
\end{abstract}

\section{INTRODUCTION}

Miniaturised satellites such as CubeSat nanosatellites have become ever more prevalent in recent years. The primary advantage of these nanosatellites is their low cost, which not only provides a low barrier to space entry but also affords redundancy and reduces mission risk by distributing functionality. For example, QB50 (Ref. 1) is a collaborative mission between international universities that was launched into orbit in 2017, consisting of a constellation of 50 CubeSats tasked with studying the lower thermosphere and re-entry research. Spacecraft travelling through the thermosphere in low Earth orbit experiences drag, ${ }^{2}$ which inevitably slows them down, shortening their operational lifetime before re-entry. Options to mitigate this include designing a minimum drag spacecraft, or the implementation of an onboard propulsion system. ${ }^{3,4}$ Such a propulsion system must be designed around volume, mass, and power budgets of the nanosatellite. Restrictions on hazardous and volatile propellants and advances in electric thruster technologies have seen electric propulsion supersede chemical propulsion as a more viable and attractive means to achieve orbital station-keeping and trajectory control manoeuvres on nanosatellites.

Some examples of low power microthruster candidates include resistojets, ${ }^{5,6}$ arcjets, ${ }^{7,8}$ and hollow cathode thrusters. ${ }^{9,10}$ Resistojets heat propellant by means of a resistively heated chamber or element but typically have a long warm up time and inefficient thermal conductance. Arcjets heat propellant by passing it through a high current electric discharge, which apart from the listed example, typically require power on the order of kiloWatts. In hollow cathodes, a heating element is used to increase the temperature of a low work function electron emitter insert in the cathode tube to temperatures required for thermionic emission. A plasma is created in the tube, which heats the insert to maintain the emission temperature. However, ion bombardment is a concern as sputtering damages the insert and reduces the lifetime of the thruster. ${ }^{11}$

Pocket Rocket (PR) is a radiofrequency (RF) plasma electrothermal microthruster currently under development by the Space Plasma, Power and Propulsion Laboratory at The Australian National University. ${ }^{12}$ At the core of $\mathrm{PR}$ is an annular RF powered electrode made of copper, situated coaxially around an alumina $\left(\mathrm{Al}_{2} \mathrm{O}_{3}\right)$ cylindrical refractory tube discharge chamber (length $18 \mathrm{~mm}$ and inner diameter $4.2 \mathrm{~mm}$ ). Cold Ar gas from an onboard miniaturised propellant subsystem ${ }^{13-15}$ is flowed through the discharge chamber, typically at a rate of $100 \mathrm{SCCM}$ or $2.97 \times 10^{-6} \mathrm{~kg} \mathrm{~s}^{-1}$. A sinusoidal RF waveform, typically at $13.56 \mathrm{MHz}$ and $300 \mathrm{~V}$ amplitude, is supplied to the powered electrode by a miniaturised RF subsystem on a printed circuit board, ${ }^{15,16}$ or an RF generator and impedance matching network in a laboratory environment. ${ }^{17}$ Using only $\lesssim 10 \mathrm{~W}$ of power, a weakly ionised plasma is ignited in the discharge chamber. The propellant gas is heated to a high temperature by the plasma, ${ }^{18-22}$ producing thrust forces on the order of $\sim 1 \mathrm{mN}$.

$\mathrm{PR}$ is similar to arcjets, where propellant is heated volumetrically and directly by a plasma. Like in resistojets, the discharge chamber wall in PR heats up after a period of operation and acts as a source of thermal energy even after the $\mathrm{RF}$ power is terminated. On the other hand, unlike resistojets and hollow cathodes which require time to warm up, plasma breakdown in PR occurs on a $\sim \mu$ s time scale; the propellant reaches target temperatures on a time scale of $\sim 1 \mathrm{~s}$, and thermal equilibrium is attained with the discharge chamber wall on the order of $\sim 10 \mathrm{~s} .^{20,22} \mathrm{PR}$ has additional advantages over hollow cathodes, as the RF powered electrode is shielded by a dielectric discharge chamber wall which protects it from damage. Instead, ion bombardment onto the discharge 
chamber wall is favourable as it creates high energy secondary electrons which sustain a gamma mode discharge. The ion density peaks in the middle of the discharge, promoting volumetric heating of the propellant via ion-neutral charge exchange collisions. $^{22}$ Conductive losses to the walls are thus minimised, thereby retaining the thermal energy beneficial for thrust.

\section{COMPUTATIONAL FLUID DYNAMICS (CFD)-PLASMA SIMULATION MODEL}

In a terrestrial laboratory, experimental instruments like the pendulum thrust balance are not sufficiently sensitive for accurately measuring such small forces, especially when the $\mathrm{RF}$ and gas feed systems have rigid attachments to the vacuum chamber, ${ }^{23,24}$ or for transient or pulsed operation. ${ }^{25}$ To satisfy this demand, a computational fluid dynamics (CFD) simulation model of PR has been developed ${ }^{26}$ using the commercial CFDACE + multiphysics package, which produced calculations for cold gas thrust that very closely matched experimental results for a version of $\mathrm{PR}$ operating with $\mathrm{Xe} .{ }^{24}$ The CFD simulation technique is designed to accurately model the cold gas operation of $\mathrm{PR}$ in both pressurised and vacuum environments by taking advantage of flow velocity choking, a compressible flow effect where the flow conditions upstream become insensitive to the flow conditions downstream under specific criteria.

Presently, the simulation technique has been expanded to include the modelling of the RF Ar discharge in PR. The CFD-plasma simulations feature six species: $\operatorname{Ar}, \operatorname{Ar}\left(4 s_{m}\right)$, $\operatorname{Ar}\left(4 s_{r}\right), \operatorname{Ar}(4 p), \mathrm{Ar}^{+}$, and $\mathrm{e}^{-}$, with a total of 29 reactions. $^{27}$ Further details of the CFD-plasma model and simulation procedures have been extensively documented in the previous publications. $^{22,26,27}$ The present model is a significant improvement to past models, ${ }^{28}$ with not only a more complete chemical reaction set but also a more comprehensive material properties database, as well as more physically accurate fluid, heat, electric, and plasma boundary conditions. The full axisymmetric PR mesh now includes the powered electrode, insulation, and structure solid regions in addition to the original PR mesh used in the cold gas performance study. ${ }^{26}$

This paper presents CFD-plasma simulations of three $P R$ geometrical variations, two of which are introduced in the previous cold gas performance study: ${ }^{26}$ the original cylindrical discharge chamber $(P R-O)$, the constricted nozzle $(P R-C)$, and the third is a newer improved prototype featuring a sculpted converging-diverging nozzle $(P R-N)$. These $P R$ geometries are shown in Fig. 1. The core regions of $P R$ are similar across the three geometrical variations and are colour coded according to the material: the discharge chamber wall (yellow, alumina), powered RF electrode (brown, copper), insulation (teal, Macor), and structure (grey, aluminium). Ar gas flows into PR from an inlet in the corner of the plenum, through the discharge chamber, and exits into the downstream region (cropped to fit in Fig. 1). In Fig. 1(a), $n_{\mathrm{i}}(t)$ peaks in the middle of the discharge chamber under the powered electrode. The plasma is seen to extend into the plenum and downstream regions and visually matches experimental observations. ${ }^{12,29}$

$\mathrm{PR}-\mathrm{N}$ is the culmination of an iterative design process targeting effective plasma confinement within the discharge
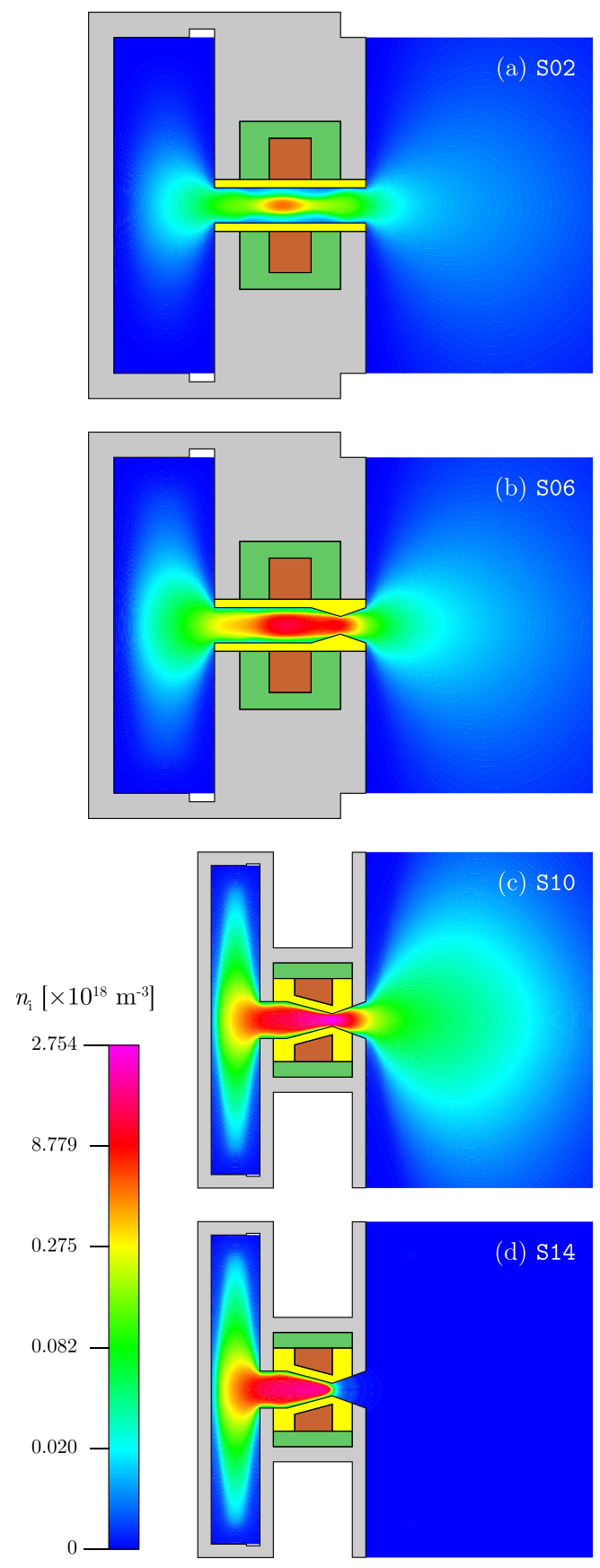

FIG. 1. 2D colour map of the cycle average ion density $n_{\mathrm{i}}$ in the cross section of the different geometrical variations of PR. The colour scale is logarithmic with the yellow region of the spectrum representing $1 / 10$ of the full scale. The propellant inlet is the notch in the corner of the plenum, and the flow direction is from left to right. From top to bottom: (a) PR-O (S02), (b) $\mathrm{PR}-\mathrm{C}(\mathrm{S} 06)$, and (c) PR-N (S10) operating in a background pressure of $p_{0}=0.349$ Torr, and (d) PR $-\mathrm{N}$ (S14) demonstrating plasma confinement when operating in $p_{0}=0.1$ Torr. The solid regions are: the discharge chamber wall (yellow, $\mathrm{Al}_{2} \mathrm{O}_{3}$ ), powered $\mathrm{RF}$ electrode (brown, $\mathrm{Cu}$ ), insulation (dark green, Macor), and structure (grey, Al). PR-C.

chamber. This is advantageous as it eliminates the return ion current onto the exterior surfaces of the spacecraft, thus preventing the contamination of solar panels and interference with externally mounted instruments. Additionally, $\mathrm{PR}-\mathrm{N}$ has reduced volume and mass compared to $\mathrm{PR}-\mathrm{O}$, thus enabling better compatibility with nanosatellites. The length of the plenum has been reduced from $12 \mathrm{~mm}$ to $5.8 \mathrm{~mm}$; the 
length of the discharge chamber has been reduced from $18 \mathrm{~mm}$ to $12.6 \mathrm{~mm}$, and the diameter of the nozzle throat has been reduced from $2.1 \mathrm{~mm}$ (in PR-C) to $1.5 \mathrm{~mm}$. The angle of the converging-diverging nozzle is also optimised for thrust forces on the order of $\sim 1 \mathrm{mN} .^{30}$ Exploring design variations via CFD and CFD-plasma simulations is more time and cost effective than producing and experimentally testing multiple physical iterations. When simulation delivers a promising design, it is manufactured as a prototype, and procedures more suited for experimentation (e.g., scaling and variation of various input parameters) can proceed. Thus, work on PR progresses in a leapfrogging manner between simulation and experimentation.

The CFD-plasma simulation results presented in this paper represent the transient conditions in each PR geometry on the order of $\sim 1 \mathrm{~s}$ after plasma ignition, which is enough time for propellant to be heated by the plasma and attain local thermal equilibrium, but before any significant heating up of the discharge chamber wall or structure. As such, the performance demonstrated here represents the "instantaneous" or transient lower limit performance of PR from a cold start. Performance is expected to further increase as the temperature of the discharge chamber wall rises and attains thermal equilibrium with the heated propellant, in a scenario similar to that of the "self-heating" mode of a hollow cathode thruster.

\section{RESULTS AND DISCUSSION}

Table I lists all the CFD-plasma simulations that have been performed for the present thrust modelling study. Simulations that are the subject of previous publications (S01, S02, S03, S05, and S07) are referenced accordingly, while the rest are new simulations making their first appearance in the present paper. All the simulations are run using a propellant mass flow rate of $\dot{m}=100 \mathrm{SCCM}=2.97 \times 10^{-6}$ $\mathrm{kg} \mathrm{s}^{-1}$ of $\mathrm{Ar}$ at the inlet. The CFD-plasma simulations are run using a $V_{\mathrm{pwr}}=300 \mathrm{~V}$ amplitude sinusoidal RF waveform at $13.56 \mathrm{MHz}$ on the powered electrode. The plasma operation mode targets a power budget of $\lesssim 10 \mathrm{~W}$, keeping to values suitable for single or multiple-unit CubeSats.

The simulations in Table I are categorised into different sets according to the geometry and the background pressure boundary condition. For example, S01 to S04 are performed using the PR-O geometry. The background pressure for the cold gas simulation S01 and CFD-plasma simulation S02 is set to $p_{0}=0.349$ Torr, which is the experimentally measured static pressure in the $20 \mathrm{~L}$ vacuum chamber during operation in the laboratory. For the cold gas simulation S03, a background pressure of $p_{0}=0$ Torr is used. The validity of using a vacuum background in a CFD simulation is by virtue of a compressible flow effect called "flow velocity choking," and the accuracy of the simulation results has been previously verified. ${ }^{26}$ However, while flow velocity choking is able to stabilise fluid behaviour upstream despite undesirable conditions downstream, there is no equivalent phenomenon that can mask downstream conditions from upstream electric fields. Consequently, CFD-plasma simulations using a vacuum background pressure invariably result in divergent solutions due to the unavoidable limitations of fluid and plasma numerical techniques in a vacuum environment. Nonetheless, the validated simulations $\mathrm{S} 01, \mathrm{~S} 02$, and $\mathrm{S} 03$ provide adequate information to extrapolate the expected results (S04) for fluid behaviour during plasma operation with $p_{0}=0$ Torr. S04 is marked with an asterisk $(*)$ to indicate that the results are extrapolated from the respective referenced simulations instead of an actual CFD-plasma simulation that has been run with the listed operating conditions. The method by which

TABLE I. List of simulations presented in this paper, and a summary of the main parameters. All values for the CFD-plasma simulations are averaged over the RF cycle. The results of the "simulations" marked with an asterisk (*) are extrapolated from the respective referenced simulations, as CFD-plasma simulations cannot be performed in a vacuum environment.

\begin{tabular}{|c|c|c|c|c|c|c|c|c|c|}
\hline Sim. & Geometry & Mode & $p_{0}$ (Torr) & $p_{\mathrm{s}}($ Torr $)$ & $P(\mathrm{~W})$ & $F_{\mathrm{t}}(\mathrm{mN})$ & $F_{\mathrm{bl}}(-\mathrm{mN})$ & $I_{\mathrm{sp}}(\mathrm{s})$ & References \\
\hline S01 & $\mathrm{PR}-\mathrm{O}$ & Cold gas & 0.349 & 1.367 & $\ldots$ & 0.794 & 1.095 & 27.3 & 26 \\
\hline $\mathrm{S} 02$ & $P R-O$ & Plasma & 0.349 & 1.532 & 5.01 & 0.897 & 1.307 & 30.8 & 22,27, and 28 \\
\hline $\mathrm{S} 03$ & $\mathrm{PR}-\mathrm{O}$ & Cold gas & 0 & 1.335 & $\ldots$ & 1.363 & 0.764 & 46.8 & 26 \\
\hline $\mathrm{S} 04 *$ & $P R-O$ & Plasma & 0 & 1.496 & $\ldots$ & 1.493 & 0.943 & 51.2 & Extrapolated: $\mathrm{S} 01, \mathrm{~S} 02$, and $\mathrm{S} 03$ \\
\hline S05 & $\mathrm{PR}-\mathrm{C}$ & Cold gas & 0.349 & 2.759 & $\ldots$ & 0.851 & 1.208 & 29.2 & 26 \\
\hline S06 & $\mathrm{PR}-\mathrm{C}$ & Plasma & 0.349 & 3.408 & 13.3 & 1.116 & 1.553 & 38.3 & Present paper \\
\hline S07 & $P R-C$ & Cold gas & 0 & 2.742 & $\ldots$ & 1.425 & 0.826 & 48.9 & 26 \\
\hline S08* & $\mathrm{PR}-\mathrm{C}$ & Plasma & 0 & 3.386 & $\ldots$ & 1.818 & 1.014 & 62.4 & Extrapolated: S05, S06, and S07 \\
\hline S09 & $\mathrm{PR}-\mathrm{N}$ & Cold gas & 0.349 & 4.992 & $\ldots$ & 0.855 & 1.383 & 29.3 & Present paper \\
\hline S10 & $\mathrm{PR}-\mathrm{N}$ & Plasma & 0.349 & 6.802 & 12.4 & 1.201 & 2.008 & 41.2 & Present paper \\
\hline S11 & $\mathrm{PR}-\mathrm{N}$ & Cold gas & 0 & 4.890 & $\ldots$ & 1.432 & 1.010 & 49.1 & Present paper \\
\hline $\mathrm{S} 12 *$ & $\mathrm{PR}-\mathrm{N}$ & Plasma & 0 & 6.664 & $\ldots$ & 2.033 & 1.318 & 69.8 & Extrapolated: S09, S10, and S11 \\
\hline S13 & $\mathrm{PR}-\mathrm{N}$ & Cold gas & 0.1 & 4.963 & $\ldots$ & 1.248 & 1.194 & 42.8 & Present paper \\
\hline S14 & $\mathrm{PR}-\mathrm{N}$ & Plasma & 0.1 & 6.380 & 12.0 & 1.607 & 1.591 & 55.2 & Present paper \\
\hline S15 & $\mathrm{PR}-\mathrm{N}$ & Cold gas & 0 & 4.890 & $\ldots$ & 1.432 & 1.010 & 49.1 & Same as S11 \\
\hline $\mathrm{S} 16^{*}$ & $\mathrm{PR}-\mathrm{N}$ & Plasma & 0 & 6.286 & $\ldots$ & 1.821 & 1.357 & 62.5 & Extrapolated: S13, S14, and S15 \\
\hline S17 & $\mathrm{PR}-\mathrm{N}$ & Plasma & 0.349 & 6.560 & 10.6 & 1.143 & 1.920 & 39.2 & $\mathrm{~S} 10, \mathrm{SEEC}=0.05$ \\
\hline $\mathrm{S} 18 *$ & $\mathrm{PR}-\mathrm{N}$ & Plasma & 0 & 6.427 & $\ldots$ & 1.942 & 1.268 & 66.7 & Extrapolated: S09, S10, and S17 \\
\hline S19 & $\mathrm{PR}-\mathrm{N}$ & Plasma & 0.1 & 6.165 & 8.75 & 1.525 & 1.548 & 52.3 & $\mathrm{~S} 14, \mathrm{SEEC}=0.05$ \\
\hline $\mathrm{S} 20^{*}$ & $\mathrm{PR}-\mathrm{N}$ & Plasma & 0 & 6.074 & $\ldots$ & 1.735 & 1.320 & 59.6 & Extrapolated: S13, S14, and S19 \\
\hline
\end{tabular}


this is done is discussed later in the text, along with commentary addressing the accuracy of the method.

The above description also applies to the following sets for PR-C (S05 to S08) and PR-N (S09 to S12 and S13 to S16). For the CFD-plasma simulation S14, the background pressure $p_{0}=0.1$ Torr is set to the lowest possible value that produces a converged result. The same $p_{0}=0.1$ Torr is used for the cold gas simulation S13 to provide a direct comparison to S14. Similarly, the results of S16 are extrapolated from the respective referenced simulations. S15 is the same as $\mathrm{S} 11$ but is repeated in this set for consistency.

Finally, S17 and S19 are run using the same operating conditions as S10 and S14, but with the secondary electron emission coefficient (SEEC) set to 0.05 instead of $0.1{ }^{28} \mathrm{~S} 18$ and $\mathrm{S} 20$ are likewise extrapolated from the respective referenced simulations. While the simulation results of $P R-O$ using $\mathrm{SEEC}=0.1$ are in good agreement with experimental measurements, there is some indication that a lower SEEC is more appropriate at the slightly higher power drawn by the nozzle geometry variations of $\mathrm{PR}$. These four simulations are performed to examine the effects of a lower SEEC on the ion density, the neutral gas heating, and the resultant thrust performance.

The PR-O simulations S01 to $\mathrm{SO3}$ have previously been rigorously verified against experimental results. ${ }^{22,26,31}$ The PR-C and PR-N simulations S05 to S20 use the PR-O simulations as a foundation and are run using the same simulation technique. The only change is the use of 120 timesteps per RF cycle for the PR-N simulations instead of the original 60 time-steps per RF cycle, in order to implement a lower $p_{0}$ and also to manage the stronger coupling between the fluid and plasma components in the discharge chamber due to the narrower nozzle throat. Additionally, the higher static pressures in $\mathrm{PR}-\mathrm{N}$ allow local thermal equilibrium to be attained quicker than in $\mathrm{PR}-\mathrm{O}$, and simulation convergence is also achieved earlier.

In addition to the simulation number and input parameters: geometry, operation mode, and background pressure $p_{0}$, Table I also lists the output parameters: stagnation pressure $p_{\mathrm{s}}$ in the plenum, the cycle average RF power draw $P$ of the $\mathrm{RF}$ discharge (for the performed CFD-plasma simulations only), the thrust force $F_{\mathrm{t}}$, the boundary layer friction force $F_{\mathrm{bl}}{ }^{26}$ and the specific impulse $I_{\mathrm{sp}}$. The simulation results are discussed in Secs. III A-III F detailing the plasma, temperature, velocity, and thrust characteristics of each PR geometry. Comparisons are made to the cold gas operation mode to envisage the instantaneous or pulsed plasma operation performance of $P R$.

\section{A. Plasma}

Figures 2 and 3 plot the ion density along the central (horizontal) $z$-axis for $\mathrm{S} 02, \mathrm{~S} 06, \mathrm{~S} 10$, and $\mathrm{S} 14$ in linear scale. The solid lines denote the cycle average ion density $n_{\mathrm{i}}$ (shown earlier in Fig. 1), while the lighter coloured lines (60 lines for $\mathrm{PR}-\mathrm{O}$ and $\mathrm{PR}-\mathrm{C}$ and 120 lines for PR-N) represent the variation of $n_{\mathrm{i}}(t)$ at each time-step during the RF cycle. Vertical dashed lines divide the plots into three sections from left to right: plenum, discharge chamber, and the downstream region.

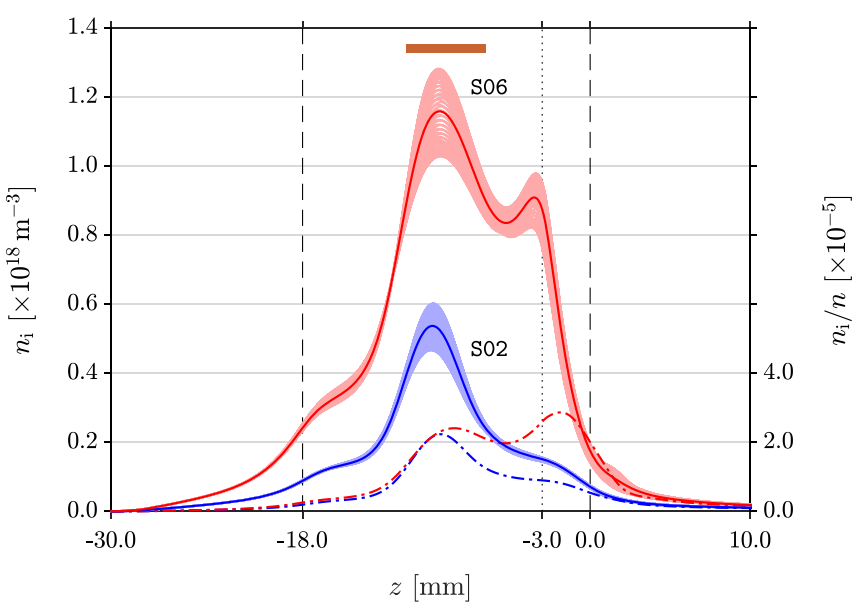

FIG. 2. Temporally varying ion density $n_{\mathrm{i}}(t)$ along the $z$-axis for PR-O S02 (blue) and PR-C S06 (red). The solid lines denote the cycle average ion density $n_{\mathrm{i}}$, shown earlier in Figs. 1(a) and 1(b). The dashed-dotted lines denote the cycle average ionisation fraction $n_{\mathrm{i}} / n$ (right vertical axis). $-30 \mathrm{~mm} \leq z$ $<18 \mathrm{~mm}$ is the plenum, while $-18 \mathrm{~mm} \leq z \leq 0 \mathrm{~mm}$ is the discharge chamber. The nozzle throat in PR-C is located at $z=-3 \mathrm{~mm}$. The brown bar at the top shows the location of the powered electrode at $-11.5 \mathrm{~mm} \leq z \leq-6.5 \mathrm{~mm}$.

A vertical dotted line shows the location of the nozzle throat for PR-C in Fig. 2 and PR-N in Fig. 3.

The $n_{\mathrm{i}}(t)$ profiles track the $n_{\mathrm{i}}$ profiles closely, without any anomalous deviation from the cycle average. For PR-O and PR-C, $n_{\mathrm{i}}$ peaks underneath the powered electrode with $n_{\mathrm{i}}=5.37 \times 10^{17} \mathrm{~m}^{-3}$ at $z=-9.9 \mathrm{~mm}$ and $n_{\mathrm{i}}=1.16 \times 10^{18}$ $\mathrm{m}^{-3}$ at $z=-9.4 \mathrm{~mm}$, respectively. The position of the central peak is slightly upstream of the midpoint of the powered electrode mainly because of the negative pressure and density gradient along the discharge chamber. The shape of the $n_{\mathrm{i}}$ profile is due to two separate ionisation modes that are dominant during the negative and positive periods of the RF cycle.

In PR, a negative self-bias ${ }^{27}$ manifests in the section of the discharge chamber wall shielding the powered electrode.

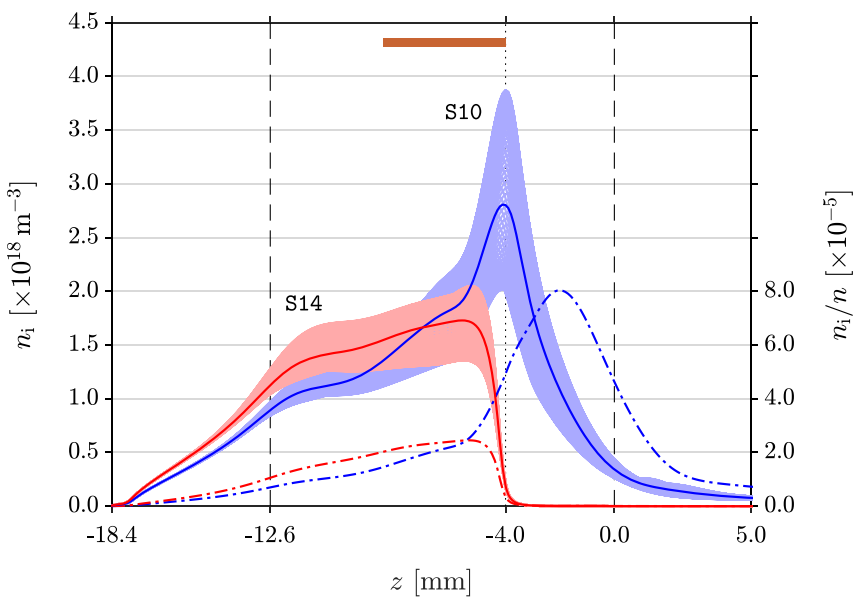

FIG. 3. Temporally varying ion density $n_{\mathrm{i}}(t)$ along the $z$-axis for PR-N S10 (blue, $p_{0}=0.349$ Torr) and S14 (red, $p_{0}=0.1$ Torr). The solid lines denote the cycle average ion density $n_{\mathrm{i}}$, shown earlier in Figs. 1(c) and 1(d). The dashed-dotted lines denote the cycle average ionisation fraction $n_{\mathrm{i}} / n$ (right vertical axis). $-18.4 \mathrm{~mm} \leq z<12.6 \mathrm{~mm}$ is the plenum, while $-12.6 \mathrm{~mm}$ $\leq z \leq 0 \mathrm{~mm}$ is the discharge chamber. The nozzle throat in PR $-\mathrm{N}$ is located at $z=-4 \mathrm{~mm}$. The brown bar at the top shows the location of the powered electrode at $-8.5 \mathrm{~mm} \leq z \leq-4 \mathrm{~mm}$. 
During the negative period of the RF cycle, ions are accelerated from the positive plasma potential towards the negative discharge chamber wall. Ion bombardment onto the surface of the discharge chamber wall results in the emission of high energy secondary electrons. These secondary electrons induce a "gamma mode" ionisation as they are accelerated through the plasma sheath into the plasma bulk, giving rise to the strong central gamma mode peak seen in the $n_{\text {i }}$ profile of $\mathrm{PR}-\mathrm{O}$ ( $\mathrm{SO2}$ ) in Fig. 2. During the positive period of the RF cycle, the self-biased section of the discharge chamber wall maintains a positive electric potential, and hence there are no secondary electrons emitted during this time. Instead, bulk electrons from upstream and downstream of the powered electrode are attracted towards the positive electric potential. Their motion in the discharge chamber induces two regions of "alpha mode" ionisation, giving rise to the two shoulder alpha mode plateaus seen on either side of the central gamma mode peak.

A similar $n_{\mathrm{i}}$ profile is observed in PR-C (S06). There is still a strong central gamma mode peak, but the downstream shoulder alpha mode plateau becomes a second peak just upstream of the nozzle throat, with $n_{\mathrm{i}}=0.91 \times 10^{18} \mathrm{~m}^{-3}$ at $z=-3.5 \mathrm{~mm}$. This is due to the high concentration of the electric field lines in the nozzle throat. During the positive period of the RF cycle, bulk electrons from the divergent section of the nozzle travel upstream towards the powered electrode along these electric field lines, inducing a high ionisation rate at the nozzle throat. To confirm that this is the case rather than a second gamma mode peak, it is necessary to examine the alpha mode ionisation rate.

Figure 4 shows a 2D colour plot of the direct ionisation rate $R_{\alpha}$ during the positive peak $(t=\pi / 2)$ of the RF cycle. Bulk electrons are responsible for ionisation events during this time as secondary electrons are only present during the negative period of the RF cycle. Figure 4(a) reflects the two shoulder alpha mode plateaus visible in the $n_{\mathrm{i}}$ profile of PR-O (S02). Correspondingly, Fig. 4(b) confirms that the second $n_{\mathrm{i}}$ peak at the nozzle throat of PR-C (S06) is due to alpha mode ionisation by bulk electrons travelling upstream, and not gamma mode ionisation by secondary electrons.

While there are significant differences between the geometries of $\mathrm{PR}-\mathrm{N}$ and $\mathrm{PR}-\mathrm{C}$, the $n_{\mathrm{i}}$ profile of S10 still bears some resemblance to that of S06. The upstream shoulder alpha mode plateau is present, as well as the downstream alpha mode peak with $n_{\mathrm{i}}=2.81 \times 10^{18} \mathrm{~m}^{-3}$ at $z=-4.1 \mathrm{~mm}$ near the nozzle throat, but the central gamma mode peak is now a diminished hump in the $n_{\mathrm{i}}$ profile. However, the ionisation fraction in S10 is roughly the same as S02 and S06 at $n_{\mathrm{i}} / n \approx 2 \times 10^{-5}$. Instead of a strong central peak, the gamma mode discharge is present along the length of the convergent section of the nozzle under the powered electrode. Like in S06, S10 has a high ionisation fraction in the divergent section of the nozzle, mainly due to the lower pressure and neutral density in the expanding plume. This is corroborated by the region of high alpha mode ionisation at the nozzle throat in Fig. 4(c), which shows the bulk electrons from the divergent section of the nozzle travelling upstream during the positive period of the RF cycle.
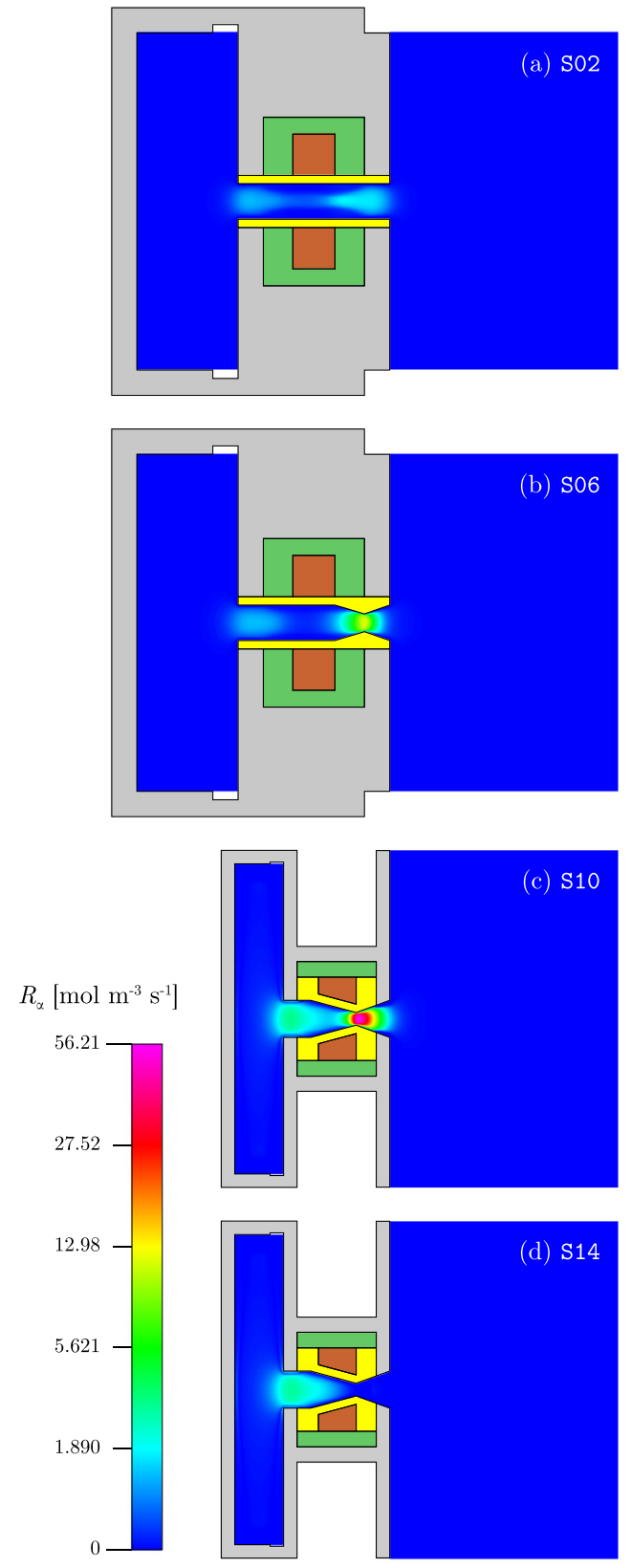

FIG. 4. 2D colour map of the direct ionisation rate $R_{\alpha}$ in (a) PR-O (S02), (b) PR-C (S06), (c) PR-N (S10), and (d) PR-N (S14) during the positive peak $(t=\pi / 2)$ of the RF cycle. The colour scale is logarithmic with the green region of the spectrum representing $1 / 10$ of the full scale.

The trapezoidal annulus shape of the powered electrode in $\mathrm{PR}-\mathrm{N}$ is designed to bring the self-biased region of the discharge chamber wall closer to the nozzle throat, while also creating a conical plasma sheath at the nozzle throat that confines the plasma upstream. The consequences of this can be seen more clearly in S14, which gives starkly different results when the background pressure is lowered to $p_{0}=0.1$ Torr. In $\mathrm{S} 14$ (Fig. 3, red) the downstream alpha mode peak at the nozzle throat disappears completely, in exchange for a slightly higher ionisation fraction throughout the discharge chamber. The gamma mode plateau merges with the upstream alpha mode plateau, forming a more uniform discharge [Fig. 1(d)], with peak $n_{\mathrm{i}}=1.73 \times 10^{18} \mathrm{~m}^{-3}$ at $z=-5.6 \mathrm{~mm}$. Although $p_{0}$ is not zero, $\mathrm{S} 14$ is demonstrative 
of how $\mathrm{PR}-\mathrm{N}$ operates in the vacuum environment of space. The accuracy of these results is substantiated later in the text.

The most important effect is the plasma confinement upstream of the nozzle throat. This occurs because the static pressure $p$ is much lower in the divergent section of the nozzle, and the width of the plasma sheath, which varies approximately inversely with $\sqrt{p},{ }^{32,33}$ becomes larger than the radius of the nozzle throat. Consequently, the plasma sheath along the discharge chamber wall merges to form a cone that terminates in the nozzle throat [Fig. 1(d)]. Thus, the discharge is restricted to the region upstream of the nozzle throat. Since there are no bulk electrons downstream of the nozzle throat, alpha mode ionisation is evidently absent in this region [Fig. 4(d)]. Similar ionisation loss behaviour has also been observed experimentally in the supersonic expanding plume of a cascade arc discharge, ${ }^{34,35}$ in which ion-neutral charge exchange collisions are responsible for enhancing recombination in the first few centimetres of the expansion. The plasma confinement is also beneficial for the convergence of the CFD-plasma simulation in the low $p_{0}$ environment of the downstream region.

Another interesting feature is the steep drop in electric potential across the plasma sheath in the nozzle throat. Ions falling through the plasma sheath are accelerated to very high velocities in the axial direction. Ion-neutral charge exchange collisions in the divergent section of the nozzle not only aid fast recombination and ensure a neutral plume but may also impart axial momentum to a small population of neutrals and be beneficial for thrust performance. ${ }^{36}$ However, due to the low ionisation fraction, this contribution and the thrust force from the remaining ions in the exhaust plume are expected to be very small relative to the main flow.

\section{B. Temperature}

The primary purpose of the plasma is to impart energy to the gas propellant in the form of heat. Many experimental techniques ${ }^{37}$ have been used to characterise neutral gas temperatures in plasmas. These include using atomic line profiles from Doppler, Stark, and van der Waals broadening, as well as rotational spectroscopy ${ }^{18,19,21}$ and laser-induced fluorescence. ${ }^{38}$ However, rotational spectroscopy becomes unreliable at low pressures, ${ }^{21,38}$ and the temperature profile in $\mathrm{PR}$ is highly nonuniform. Hence, computational modelling techniques are required.

The cycle average temperature $T$ of the background neutral Ar gas is plotted for the first four sets of simulations ( $\mathrm{S} 01$ to S16) in Figs. 5-8, comparing cold gas operation (blue lines) to plasma operation (red lines). Solid lines are for CFD and CFD-plasma simulations run with a nonzero background pressure $p_{0}$, while dashed-dotted lines are for CFD simulations run with $p_{0}=0$ Torr and CFD-plasma "simulations" extrapolated to $p_{0}=0$ Torr (S04, S08, S12, and S16).

The variation of $T(t)$ at each time-step during the RF cycle is also shown (light red lines) for the performed CFDplasma simulations (S02, S06, S10, and S14). However, it is for visual reference only, because the variation of the fluid

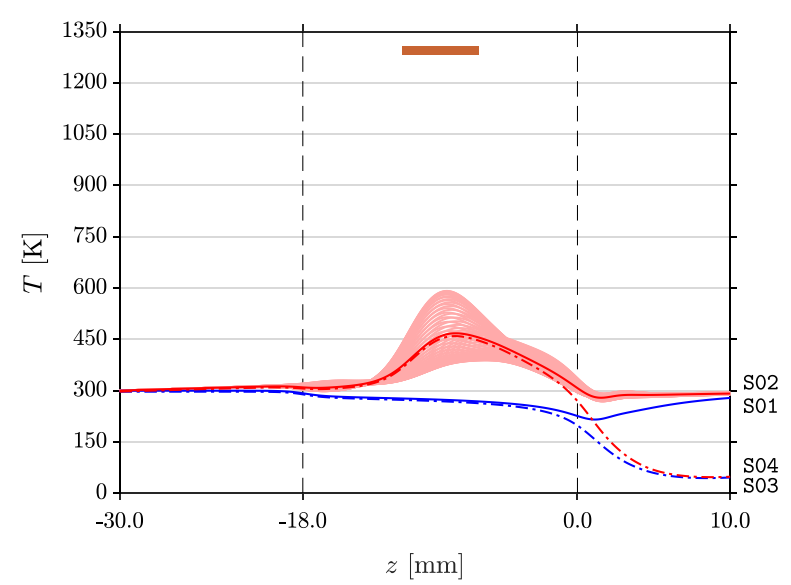

FIG. 5. Cycle average temperature $T$ along the $z$-axis for PR-O. Cold gas operation: $\mathrm{S} 01$ (blue solid line, $p_{0}=0.349$ Torr) and S03 (blue dashed dotted line, $p_{0}=0$ Torr). Plasma operation: S02 (red solid line, $p_{0}=0.349$ Torr) and $\mathrm{S} 04$ (red dashed-dotted line, $p_{0}=0$ Torr, extrapolated results).

parameters at each time-step is to a large extent a consequence of the large fluid time-step size $\left(\Delta \tau_{\mathrm{f}}=1.0 \mu \mathrm{s}\right)$ rather than an actual response to the variation of the plasma parameters over the plasma time-step size $\left(\Delta \tau_{\mathrm{p}}=1.229 \mathrm{~ns}\right)$. Hence, the fluid parameters must be averaged over the RF cycle for valid interpretation. Nonetheless, the $T(t)$ profiles provide some insight into where heating of the neutral gas by the plasma is taking place.

The extrapolated profiles are obtained using a pointwise function that transforms cold gas operation in nonzero background pressure cases to cold gas operation in $p_{0}=0$ Torr cases. Using the PR-O CFD simulations as an example, a function $f_{T}$ specifically for temperature is found such that $f_{T}\left(T_{\mathrm{S} 01}\right)=T_{\mathrm{S} 03}$, fitting all of the $T$ data points in both $\mathrm{S} 01$ (blue solid line) and S03 (blue dashed-dotted line). Note that these CFD simulations are steady state, so the $T$ profiles in $\mathrm{S}_{01}$ and $\mathrm{S} 03$ are constant in time. The same function is then applied to each of the temporally varying $T(t)$ profiles (light red lines) of the transient CFD-plasma simulation S02 and calculates the temporally varying $T(t)$ profiles of S04 (not shown in Fig. 5). $f_{T}\left(T_{\mathrm{S} 02}(t)\right)=T_{\mathrm{S} 04}(t)$ thereby gives a prediction of the $T(t)$

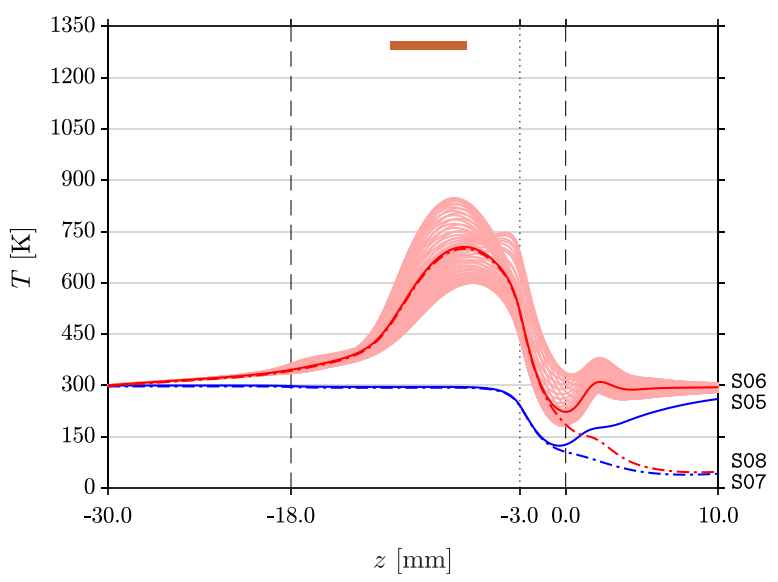

FIG. 6. Cycle average temperature $T$ along the $z$-axis for PR-C. Cold gas operation: S0 5 (blue solid line, $p_{0}=0.349$ Torr) and S07 (blue dashed dotted line, $p_{0}=0$ Torr). Plasma operation: $\mathrm{S} 06$ (red solid line, $p_{0}=0.349$ Torr) and $\mathrm{S} 08$ (red dashed-dotted line, $p_{0}=0$ Torr, extrapolated results). 


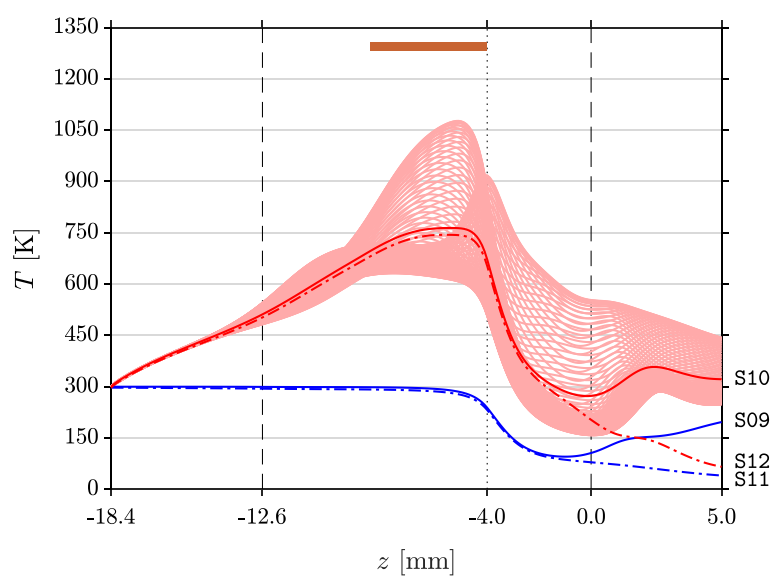

FIG. 7. Cycle average temperature $T$ along the $z$-axis for $\mathrm{PR}-\mathrm{N}$. Cold gas operation: S09 (blue solid line, $p_{0}=0.349$ Torr) and S11 (blue dashed dotted line, $p_{0}=0$ Torr). Plasma operation: $\mathrm{S} 10$ (red solid line, $p_{0}=0.349$ Torr) and $\mathrm{S} 12$ (red dashed-dotted line, $p_{0}=0$ Torr, extrapolated results).

profiles in $\mathrm{PR}-\mathrm{O}$ during plasma operation in $p_{0}=0$ Torr (S04), using data from the three performed simulations in $p_{0}=0.349$ Torr $(\mathrm{S} 01, \mathrm{~S} 02$, and S03). Finally, the calculated $T(t)$ profiles of $\mathrm{S} 04$ are averaged over the RF cycle, resulting in the $T$ profile of S04 plotted in Fig. 5 (red dashed-dotted line). The same procedure is performed for the other sets of simulations, with unique $f_{T}$ for each set. The procedure also works for other fluid parameters like density, pressure, and velocities, with functions specific to each parameter, e.g., $f_{u_{z}}\left(u_{z, \mathrm{~S} 01}(t)\right)=u_{z, \mathrm{~S} 03}(t) \Rightarrow f_{u_{z}}\left(u_{z, \mathrm{~S} 02}(t)\right)=u_{z, \mathrm{~S} 04}(t)$. Plasma parameters cannot be extrapolated as the cold gas simulations do not contain such information.

Figure 5 plots the $T$ profiles of PR-O. SO1 (blue solid line) and S03 (blue dashed-dotted line) are the cold gas operation cases. The initial temperature of the neutral gas propellant is $T=300 \mathrm{~K}$ at the front plenum wall $(z=-30 \mathrm{~mm})$. There is a slight drop in $T$ as the propellant enters the discharge chamber $(z=-18 \mathrm{~mm})$. This is due to a slight acceleration of the flow called the Venturi effect, caused by the sudden constriction of the flow diameter from $40 \mathrm{~mm}$ in the plenum to $4.2 \mathrm{~mm}$ in the discharge chamber.

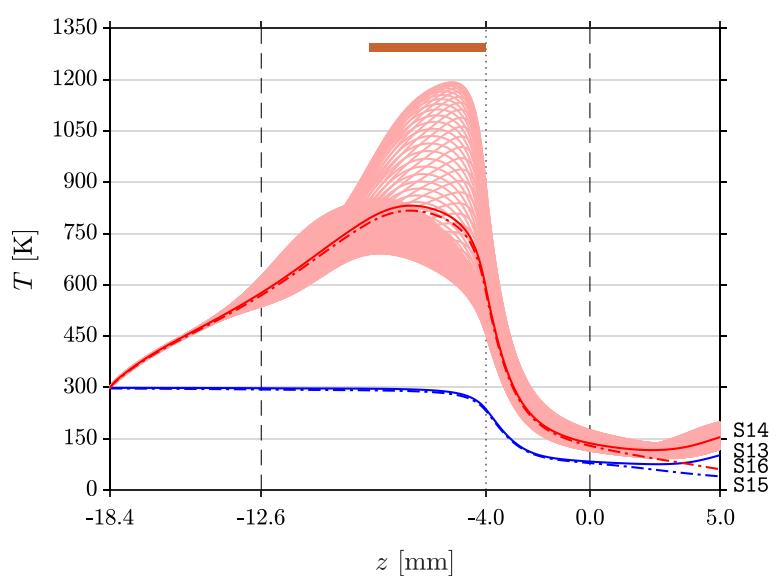

FIG. 8. Cycle average temperature $T$ along the $z$-axis for PR-N. Cold gas operation: S13 (blue solid line, $p_{0}=0.1$ Torr) and S15 (blue dashed dotted line, $p_{0}=0$ Torr). Plasma operation: $\mathrm{S} 14$ (red solid line, $p_{0}=0.1$ Torr) and $\mathrm{S} 16$ (red dashed-dotted line, $p_{0}=0$ Torr, extrapolated results).
Along the plenum and discharge chamber, $T$ falls monotonically as the propellant is accelerated by the pressure gradient bridging the stagnation pressure $p_{\mathrm{s}}$ in the plenum (see Table I) to $p_{0}$ downstream. There is another drop in $T$ as the propellant exits the discharge chamber and expands into the lower pressure environment downstream. In $S 01, T$ returns to thermal equilibrium with the ambient gas at $p_{0}=0.349$ Torr and $T=300 \mathrm{~K}$. In S03 on the other hand, $T$ continues to fall as the propellant expands into vacuum. The accuracy of the fluid parameters a certain distance beyond the discharge chamber exit cannot be guaranteed for the CFD simulations run with $p_{0}=0$ Torr. This is discussed in more detail in Sec. III C.

During plasma operation, the $T$ profile of S02 (red solid line) rises slightly in the plenum due to the presence of a weak plasma in the region. The slight drop at $z=-18 \mathrm{~mm}$ is still present, but $T$ quickly rises in the discharge chamber due to heating by the plasma. The peak temperature is attained with $T=467.7 \mathrm{~K}$ at $z=-8 \mathrm{~mm}$, which is slightly downstream of the $n_{\mathrm{i}}$ peak. This is expected as thermalisation happens while the propellant is flowing downstream at significant velocities. The difference in temperature between plasma operation ( $\mathrm{SO2}$ ) and cold gas operation ( $\mathrm{SO1}$ ) is $\Delta T=+195.9 \mathrm{~K}$ at this location.

The extrapolated $T$ profile of S04 (red dashed-dotted line, $p_{0}=0$ Torr) mostly follows that of S02 (red solid line, $p_{0}=0.349$ Torr) in the plenum and discharge chamber. This is the expected result since the flow conditions upstream of the sonic surface ${ }^{39}$ are insensitive to the flow conditions downstream due to flow velocity choking. ${ }^{26}$ Past the discharge chamber exit, the $T$ profile of S04 transitions follows that of S03 (blue dashed-dotted line). As such, it deviates from the verifiable $T$ profile of $\mathrm{S} 02$, and there is indeterminate uncertainty regarding the accuracy of the data in the downstream region. Nonetheless, only the results up to $z \leq 0 \mathrm{~mm}$ are necessary for characterising the performance of $\mathrm{PR}$, and the deviation is small within the discharge chamber.

Figure 6 plots the $T$ profiles of PR-C, and the main features are quite similar to those of PR-O (Fig. 5). In PR-C, the pressure gradient is mostly dropped at the nozzle throat, and so the temperature drop associated with the acceleration of the propellant occurs at the nozzle throat $(z=-3 \mathrm{~mm})$. The temporally varying $T(t)$ profiles of S06 (light red lines) reveal a peak that corresponds to the position of the central gamma mode peak, as well as a second peak that corresponds to the downstream alpha mode peak at the nozzle throat. The combined heating from these two locations results in a $T$ profile (red solid line) that peaks in between the powered electrode and the nozzle throat. The maximum temperature is attained at $T=704.9 \mathrm{~K}(\Delta T=+409.5 \mathrm{~K})$ at $z=-6.6 \mathrm{~mm}$. The larger increase in temperature is primarily due to higher $n_{\mathrm{i}}$ and also constitutes a higher power draw. At the discharge chamber exit, the $T$ profile shows a large dip, which indicates significant overexpansion of the exhaust plume. This is expected, as the nozzle geometry is more suited for operating in a near-vacuum environment rather than at $p_{0}=0.349$ Torr. There is a small bump following the dip as the fast expanding exhaust plume encounters the static background gas before reaching thermal equilibrium. 
As for S08 (red dashed-dotted line), the $T$ profile follows that of S06 almost exactly in the discharge chamber. There is less deviation in the results upstream of the nozzle throat, since the nozzle throat provides a more well defined vena contracta. This is advantageous as there is little to no error or uncertainty in the $f_{T}$ (as well as the functions for other fluid parameters), and all the extrapolated fluid parameters are accurate in that region. However, there is still a similar amount of deviation near the discharge chamber exits in $\mathrm{PR}-\mathrm{C}$ as in $\mathrm{PR}-\mathrm{O}$.

Figure 7 plots the $T$ profiles of PR-N (S09 to $\mathrm{S} 12$ ). While the cold gas operation S09 (blue solid line) and S11 (blue dashed-dotted line) look very similar to those of PR-C (Fig. 6), there is a striking difference in the $T$ profile of S10 during plasma operation. The temperature in the plenum has increased significantly and rises almost linearly along the $z$-axis. This creates a $T$ profile in the shape of a wedge instead of a peaked profile like those seen earlier in PR-O and PR-C. Closer inspection of the temporally varying $T(t)$ profiles reveals features very similar to the shape of the $n_{\mathrm{i}}$ profile (Figs. 1 and 3, blue solid line). In the discharge chamber, there is a plateau in the $T(t)$ profiles, followed by a peak, and then a second peak. The plateau in the $T(t)$ profiles is more evident in $\mathrm{PR}-\mathrm{N}$ as the upstream alpha mode ionisation is significantly higher than in PR-O and PR-C (see Fig. 4). While the top of the $T$ profile is almost flat, the maximum temperature $T$ $=764.0 \mathrm{~K}$ is attained at $z=-5.4 \mathrm{~mm}$, slightly upstream of the nozzle throat. This gives a temperature difference of $\Delta T=+474.9 \mathrm{~K}$.

An interesting result is the large variation in the $T(t)$ profiles in the divergent section of the nozzle. This is mainly due to the cyclic heating by the alpha mode discharge at the nozzle throat. The variation is much larger than in PR-C (Fig. 6, light red lines) as it directly corresponds to $n_{\mathrm{i}}(t)$ (Fig. 3 , light blue lines) and the alpha mode ionisation [Fig. 4(c)] in the nozzle throat. The large variation in the $T(t)$ profiles persists in the downstream region but as a whole produces the same overexpansion feature in $T$ seen earlier in PR-C. The temporal variation in $T(t)$ together with the overexpansion results in a larger deviation of the $T$ profile of S12 (red dashed dotted line) from that of S10 at the discharge chamber exit.

The fourth set of simulations performed with $P R-N$ (Fig. 8, S13 to S16) eliminates all of the issues mentioned earlier: the deviation of the extrapolated results from the results of the performed CFD-plasma simulation, the overexpansion of the exhaust plume exiting into a high $p_{0}$ environment, and the inability to accurately model the plasma operation of $P R$ in a vacuum environment. For the cold gas operation cases S13 (blue solid line) and S15 (blue dasheddotted line), the $T$ profiles are very similar to that of S09 and S11 (Fig. 7), except that they deviate after $z=0 \mathrm{~mm}$ rather than before. This is evidence that $p_{0}=0.1$ Torr, which is sufficiently high for fluid numerical techniques to be valid, is at the same time sufficiently low for emulating the fluid parameters of $\mathrm{PR}-\mathrm{N}$ operating in vacuum, with adequate accuracy at least up to $z \leq 0 \mathrm{~mm}$.

The $T(t)$ profiles of $\mathrm{S} 14$ (light red lines) differ from that of S10 (Fig. 7) even though the same geometry is used. This is because of the distinctly different discharge characteristics in $\mathrm{PR}-\mathrm{N}$ when operating in a background pressure of $p_{0}=0.1$ Torr. The upstream plateau and downstream peak features in the $T(t)$ profiles are replaced by a single lower but wider peak corresponding to the location of the alpha mode ionisation [Fig. 4(d)]. The overlap of the alpha mode and gamma mode ionisation regions results in a much higher peak in $T(t)$ under the powered electrode. The absence of plasma in the divergent section of the nozzle [Figs. 1(d) and 3, light red lines) has also resulted in a stable temperature in neutral exhaust plume.

Overall, the cycle average $T$ profile of S14 (solid red lines) still has the shape of a wedge, but with steeper gradients and a rounded top. The maximum temperature is $T=831.6 \mathrm{~K}$, attained at $z=-6.9 \mathrm{~mm}$. The difference in temperature compared with cold gas operation is $\Delta T=+537.2 \mathrm{~K}$, which is the largest increase recorded so far. The $T$ profile also does not show overexpansion at the discharge chamber exit, indicating that $p_{0}=0.1$ Torr is low enough to mimic vacuum expansion of the propellant in $\mathrm{PR}-\mathrm{N}$.

Because the $T$ profiles of S13 and S15 are almost identical throughout PR-N, the extrapolation function $f_{T}$ makes very little adjustments to the $T$ profile of S16 (red dasheddotted lines) up to $z \leq 0 \mathrm{~mm}$. Essentially, this means that S14 performed with $p_{0}=0.1$ Torr can be considered to be a proxy for obtaining an equivalent CFD-plasma model of $\mathrm{PR}-\mathrm{N}$ operating in $p_{0}=0$ Torr, thereby overcoming the limitations of the fluid and plasma numerical techniques in a vacuum environment. Nonetheless, extrapolation of S16 is performed for consistency and comparison to other extrapolated results (S04, S08, and S12).

\section{Knudsen number}

Another reason for addressing the problem via CFDplasma simulation is that the alternative via theoretical means brings about particular difficulties as the flow is in the slip regime, where the Navier-Stokes equations are only valid in the main flow and not in the boundary layer. In this regime, it is necessary to use the slip boundary condition, which requires a full CFD treatment. ${ }^{26}$

The slip regime is characterised by a Knudsen number in the range of $0.01 \lesssim \mathrm{Kn} \lesssim 0.1$. $\mathrm{Kn}$ is a dimensionless parameter given by the ratio of the mean free path of a molecule $\lambda$ to the characteristic length of the flow system $L$. The mean free path is defined as

$$
\lambda=\frac{k_{\mathrm{B}} T}{\sqrt{2} \pi \sigma^{2} p},
$$

where $k_{\mathrm{B}}=1.381 \times 10^{-12} \mathrm{~J} \mathrm{~K}^{-1}$ is the Boltzmann constant, while $T$ and $p$ are the local temperature and static pressure, respectively. Note that the $\lambda$ uses the kinetic description with the Lennard-Jones collision diameter $\sigma$ instead of the fluid description with the fluid dynamic viscosity, as the former definition preserves its accuracy even in high Kn regimes. The characteristic length of the flow system is half of the shortest dimension in each region. In the plenum, $L$ is half the length; in the discharge chamber, $L$ is the radius; in the 


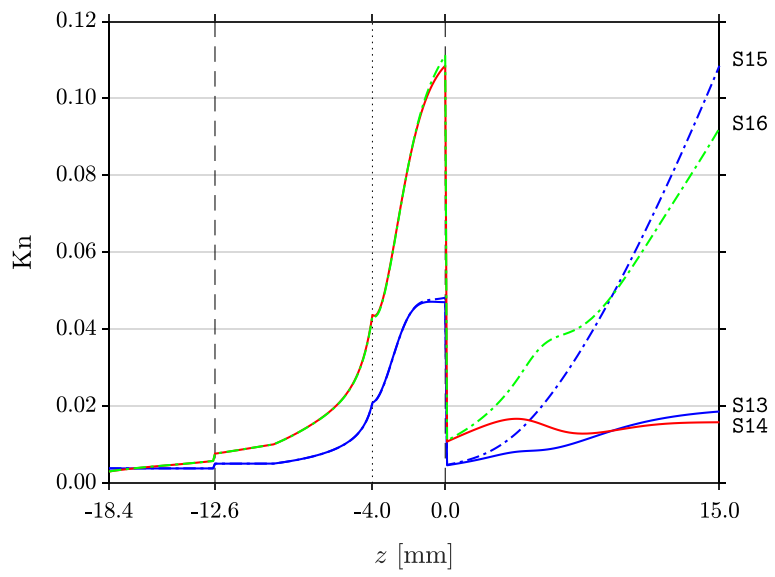

FIG. 9. Knudsen number Kn for S13 (blue solid line), S14 (red solid line), S15 (blue dashed-dotted line), and S16 (green dashed-dotted line). Flow in $\mathrm{PR}-\mathrm{N}$ is in the slip regime $(0.01 \leqq \mathrm{Kn} \lesssim 0.1)$.

downstream region, $L=22.5 \mathrm{~mm}$ (radius of the glass expansion tube in the experimental setup ${ }^{12}$ ) is used as a worst case scenario. Figure 9 plots $\mathrm{Kn}$ for the fourth set of simulations using $\mathrm{PR}-\mathrm{N}$ as an example, demonstrating that most of the flow is in the slip regime, and hence simple theoretical calculations are inadequate for providing an accurate understanding of the flow characteristics in PR-N.

CFD and CFD-plasma techniques on the other hand are well suited for treating flows in the slip regime. In particular, the low $\mathrm{Kn}$ in the downstream region of S13 and S14 confirms that the fluid and plasma results are valid at $p_{0}=0.1$ Torr. Although $\mathrm{Kn}$ is at the upper limit of the slip regime in $\mathrm{S} 15$, the results from this CFD simulation are almost identical to that of S13, indicating that they are also valid and accurate to a high degree. Since the extrapolation from S14 to S16 is very close to the original CFD-plasma simulation results, S16 is therefore valid and accurate as well, up to $z \leq 0 \mathrm{~mm}$. As Kn quickly rises in the downstream region for $\mathrm{S} 15$ and $\mathrm{S} 16$, there is a limited distance past the discharge chamber exit that the CFD and CFD-plasma results remain accurate. In practice, all results past $z>0 \mathrm{~mm}$ are discarded to preserve the integrity of the data and the rigour of the method.

\section{Velocity}

The primary purpose of heating in $P R$ is to increase the exit velocity of the propellant. There is no easy method to experimentally measure the velocity profile of the propellant inside the discharge chamber due to its geometry and small physical dimensions. Again, the only solution is by computational means, with a full treatment of boundary layer effects which are dominant in the slip regime.

Figures $10-13$ plot the cycle average axial velocity $u_{z}$ profiles for the first four sets of simulations ( $\mathrm{SO1}$ to S16), comparing cold gas operation (blue lines) to plasma operation (red lines), and also comparing operating in a nonzero $p_{0}$ environment (solid lines) for operating in a vacuum environment with $p_{0}=0$ Torr (dashed-dotted lines). During plasma operation, the $u_{z}$ profiles (red solid lines) clearly show a marked increase in the acceleration of the propellant at the nozzle

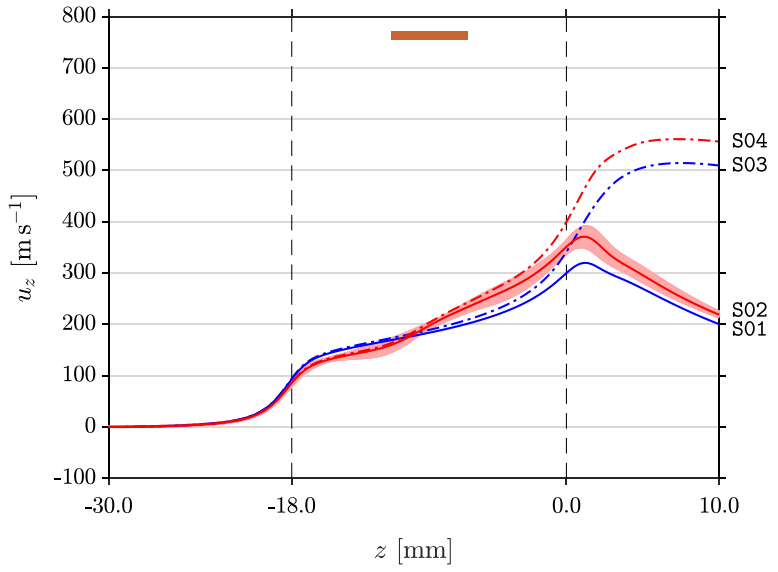

FIG. 10. Cycle average axial velocity $u_{z}$ along the $z$-axis for PR-O. Cold gas operation: $\mathrm{S} 01$ (blue solid line, $p_{0}=0.349$ Torr) and S03 (blue dashed dotted line, $p_{0}=0$ Torr). Plasma operation: $\mathrm{S} 02$ (red solid line, $p_{0}=0.349$ Torr) and S 44 (red dashed-dotted line, $p_{0}=0$ Torr, extrapolated results).

throat of PR-C (Fig. 11) and PR-N (Figs. 12 and 13). Although plasma operation raises the temperature of the propellant in PR-O (Fig. 10), the energy of the hot propellant is not effectively transformed to acceleration. Another advantage of the nozzle geometries of PR-C and PR-N is the low $u_{z}$ in the discharge chamber. The propellant takes a longer time to transit through the discharge chamber, thereby allowing more time for effective thermalisation with the hot ions through ion-neutral charge exchange collisions, ${ }^{22}$ which are also more numerous at the higher pressures in $\mathrm{PR}-\mathrm{C}$ and $\mathrm{PR}-\mathrm{N}$.

For a short time just after the negative peak $(t=3 \pi / 2)$ of the RF cycle, the temporally varying $u_{z}(t)$ profiles of $S 10$ (Fig. 12) and S14 (Fig. 13) become negative (light magenta lines) in the region of the discharge chamber just upstream of the powered electrode, while also being the highest downstream of the powered electrode. This is a strong suggestion of neutral pumping by ion-neutral charge exchange collisions $^{40,41}$ for two reasons. First, it occurs symmetrically on either side of the gamma mode discharge. Second, its occurrence is just after the peak of the gamma mode ionisation, during which a new population of hot ions has been created.

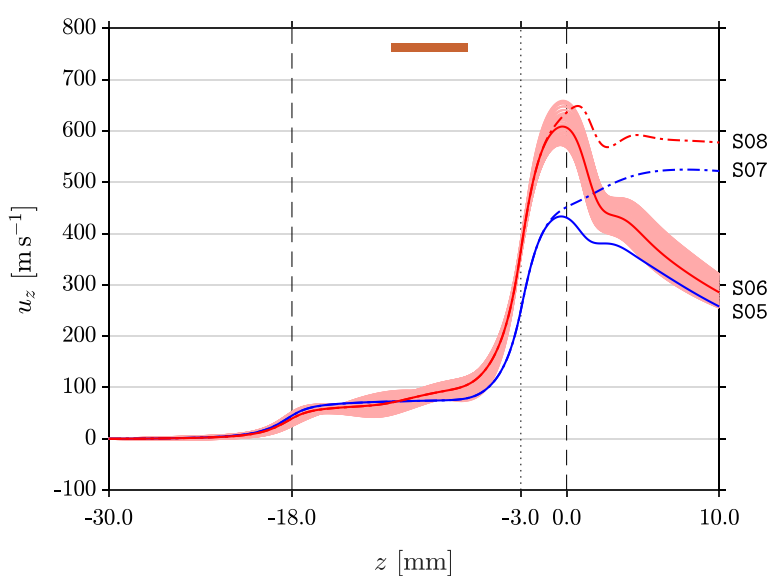

FIG. 11. Cycle average axial velocity $u_{z}$ along the $z$-axis for PR-C. Cold gas operation: S05 (blue solid line, $p_{0}=0.349$ Torr) and S07 (blue dashed dotted line, $p_{0}=0$ Torr). Plasma operation: $\mathrm{S} 06$ (red solid line, $p_{0}=0.349$ Torr) and S0 8 (red dashed-dotted line, $p_{0}=0$ Torr, extrapolated results). 


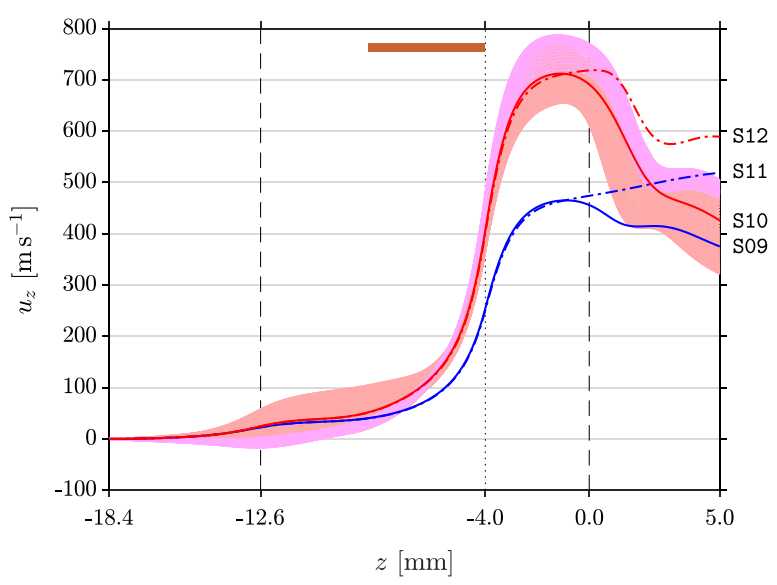

FIG. 12. Cycle average axial velocity $u_{z}$ along the $z$-axis for PR-N. Cold gas operation: $\mathrm{S} 09$ (blue solid line, $p_{0}=0.349$ Torr) and S11 (blue dashed dotted line, $p_{0}=0$ Torr). Plasma operation: $\mathrm{S} 10$ (red solid line, $p_{0}=0.349$ Torr) and $\mathrm{S} 12$ (red dashed-dotted line, $p_{0}=0$ Torr, extrapolated results).

The neutral pumping is unlikely to be due to heating by electron-neutral elastic collisions, first because the ion-neutral charge exchange collisions are far more dominant in terms of the amount of power transferred to the neutrals ${ }^{22}$ and second the time and position of the peak electron-neutral elastic collision rate do not synchronise with the neutral pumping feature in the $u_{z}(t)$ profiles.

While the $u_{z}(t)$ profiles demonstrate neutral pumping both upstream against the main flow and downstream accelerating over the main flow, the same behaviour is not perceptible in the radial direction towards the discharge chamber wall, as the radial velocity $u_{r}$ is convergent towards the central axis of the nozzle throat. In any case, the neutral pumping features (light magenta lines) do not constitute absolute evidence of the phenomenon, as the temporal variation of the $u_{z}(t)$ profiles is to a certain degree a result of the large fluid time-step size rather than an actual response to the variation of the plasma parameters over the plasma time-step size.

In all the simulations performed with $p_{0}=0.349$ Torr (Figs. 10-12; solid lines), the $u_{z}$ profiles decline rapidly as

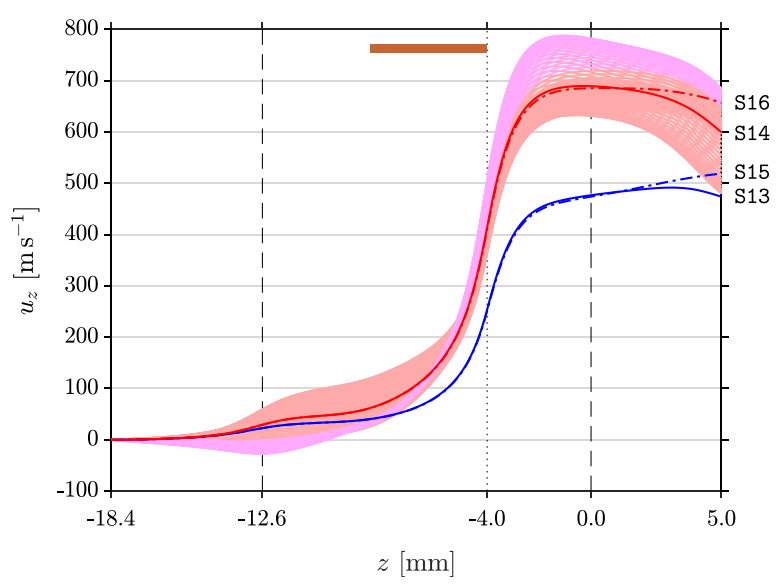

FIG. 13. Cycle average axial velocity $u_{z}$ along the $z$-axis for PR-N. Cold gas operation: S13 (blue solid line, $p_{0}=0.1$ Torr) and S15 (blue dashed dotted line, $p_{0}=0$ Torr). Plasma operation: $\mathrm{S} 14$ (red solid line, $p_{0}=0.1$ Torr) and $\mathrm{S} 16$ (red dashed-dotted line, $p_{0}=0$ Torr, extrapolated results). the exhaust plume encounters the static gas in the downstream region. For $\mathrm{PR}-\mathrm{C}$ and $\mathrm{PR}-\mathrm{N}$, the $u_{z}$ profiles already start to decrease in the divergent section of the nozzle due to overexpansion into the high background pressure. This creates an indeterminate uncertainty when extrapolating those results to $p_{0}=0$ Torr, as seen by the deviation between the solid and dashed-dotted lines. This problem is solved by using $p_{0}=0.1$ Torr instead (Fig. 13, solid lines), which produces almost identical results for $\mathrm{S} 13$ (blue solid line) and S15 (blue dashed-dotted line). Extrapolation of the $u_{z}$ profile from $\mathrm{S} 14$ (red solid line) to S16 (red dashed-dotted line) is therefore minimal. Moreover, the $u_{z}$ profile has an almost constant and flat gradient at the discharge chamber exit, indicating no overexpansion in the nozzle, and thus ensures a high degree of accuracy in the extrapolated results.

\section{E. Thrust force}

As mentioned earlier, terrestrial experimental instruments are often not sufficiently accurate for measuring thrust forces on the order of $\sim 1 \mathrm{mN}$ or thrust force variations in smaller fractions of this value. Alternatively, thrust forces are often estimated using theoretical methods that are only valid for conventional nozzles, neglecting the important slip regime boundary layer effects that are dominant at the operating conditions of the microthruster. For example, calculating the thrust force from the pressure force imbalance in the microthruster does not take into account the friction forces between the boundary layer and the wall. ${ }^{26}$ Another commonly used method is to assume that the exit velocity of the propellant is the local sound speed $c_{\mathrm{s}}=\sqrt{\gamma k_{\mathrm{B}} T / m}$, where $\gamma$ is the adiabatic index, $T$ is the local temperature of the propellant, and $m$ is the molecular mass of the propellant. If there is a nozzle, then acceleration of the propellant is assumed to be relative to the ratio of the exit area to the throat area. In reality, the sonic surface is not flat, and the exit velocity has a profile that is highly modulated by the boundary layer near the wall and cannot be simplified to a single number. Again, a full CFD treatment with the appropriate slip boundary condition is required to accurately model the flow characteristics in this $\mathrm{Kn} \lesssim 0.1$ regime.

Figures $14-17$ plot the exit cycle average axial velocity $u_{z, \text { ex }}$ profiles for the first four sets of simulations. In $\mathrm{PR}-\mathrm{O}$ (Fig. 14), the $u_{z, \text { ex }}$ profiles of the $p_{0}=0$ Torr cases (dasheddotted lines) are displaced positively relative to the $u_{z, \mathrm{ex}}$ profiles of the $p_{0}=0.349$ Torr cases (solid lines), while preserving the rounded shape. This indicates that the propellant flow is simply accelerated by a certain amount, and the width of the boundary layer at the exit is roughly the same in the four $\mathrm{PR}-\mathrm{O}$ simulations. On the other hand, there is a pronounced difference between the $u_{z, \text { ex }}$ profiles for the $p_{0}=0$ Torr (dashed-dotted lines) and $p_{0}=0.349$ Torr (solid lines) cases in PR-C (Fig. 15) and PR-N (Fig. 16). This is a sign of overexpansion in the $p_{0}=0.349$ Torr cases (solid lines). The concern here is with the accuracy of the extrapolated $u_{z, \text { ex }}$ profiles (red dashed-dotted lines), which have a large deviation from the original CFD-simulation results. In the fourth set of simulations performed with PR $-\mathrm{N}$ (Fig. 17), 


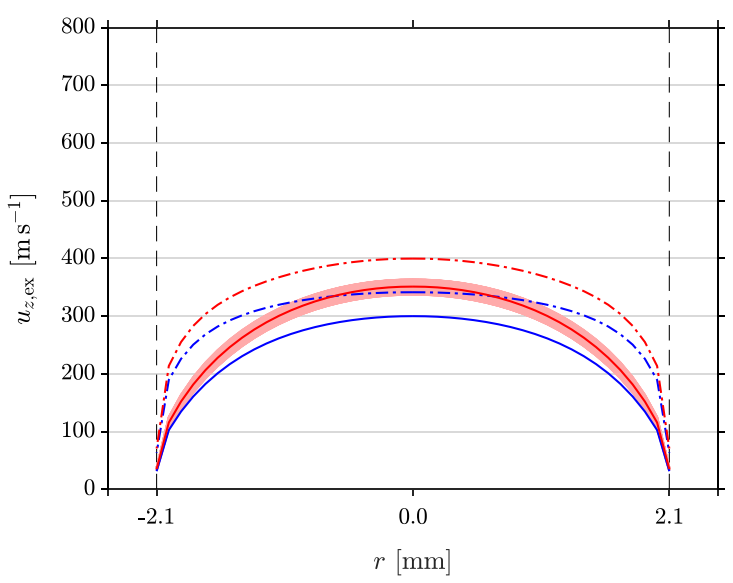

FIG. 14. Cycle average axial velocity $u_{z, \mathrm{ex}}$ across the discharge chamber exit for PR-O. Cold gas operation: $\mathrm{S} 01$ (blue solid line, $p_{0}=0.349$ Torr) and S03 (blue dashed dotted line, $p_{0}=0$ Torr). Plasma operation: S02 (red solid line, $p_{0}=0.349$ Torr) and S0 4 (red dashed-dotted line, $p_{0}=0$ Torr, extrapolated results).

the propellant is ideally expanded in both the $p_{0}=0.1$ Torr and $p_{0}=0$ Torr cases.

The $u_{z, \text { ex }}$ profiles of the nozzle geometries PR-C and PR-N (Figs. 15-17) are strongly peaked in the centre of the discharge chamber exit, as the flow on the central axis is least affected by viscosity and friction from the boundary layer. The rising portion of the $u_{z, \text { ex }}$ profiles near the wall shows that the boundary layer is dominant over a significant area of the flow cross section. Neglecting to account for this, as it often happens, inevitably results in gross overestimations of the propellant exit velocity, and therefore the estimated thrust force and other performance metrics. Trying to find the shape of the $u_{z, \text { ex }}$ profile via theoretical means is nontrivial; the fictitious slip velocity and temperature jump ${ }^{42}$ parameters at the wall must be determined using a full CFD treatment with appropriate tangential momentum and thermal accommodation coefficients. ${ }^{26}$

In the extrapolated plasma operation results, the peak exit velocity attained is: $u_{z, \mathrm{ex}}=399.7 \mathrm{~m} \mathrm{~s}^{-1}$ or Mach 3.1 in PR-O, $u_{z, \mathrm{ex}}=635.9 \mathrm{~m} \mathrm{~s}^{-1}$ or Mach 2.5 in PR-C, $u_{z, \mathrm{ex}}=718.4 \mathrm{~m} \mathrm{~s}^{-1}$

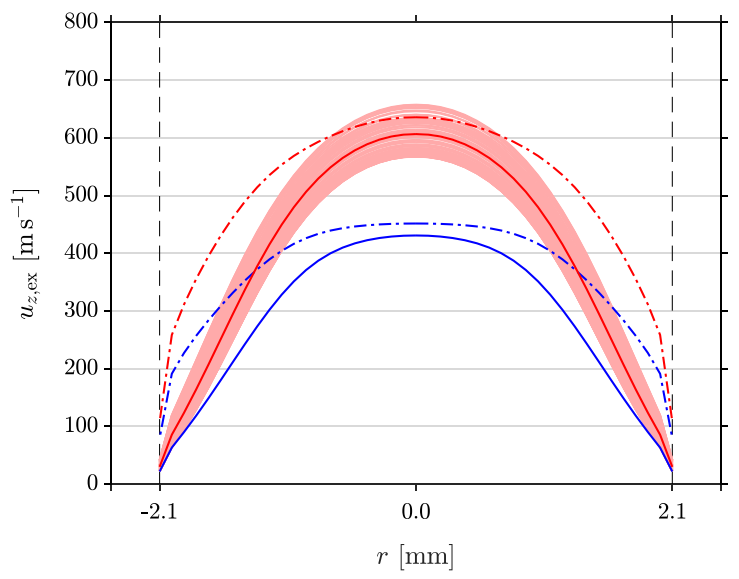

FIG. 15. Cycle average axial velocity $u_{z, \text { ex }}$ across the discharge chamber exit for PR-C. Cold gas operation: S05 (blue solid line, $p_{0}=0.349$ Torr) and S 07 (blue dashed dotted line, $p_{0}=0$ Torr). Plasma operation: S06 (red solid line, $p_{0}=0.349$ Torr) and S 08 (red dashed-dotted line, $p_{0}=0$ Torr, extrapolated results).

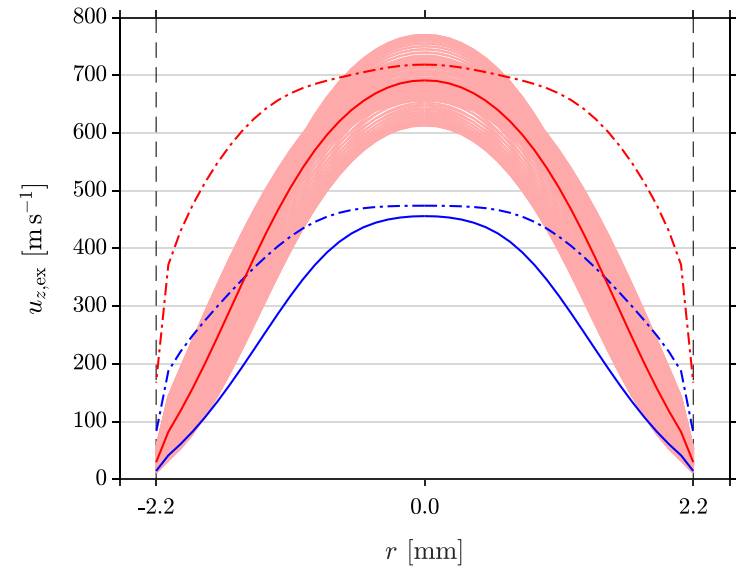

FIG. 16. Cycle average axial velocity $u_{z, \text { ex }}$ across the discharge chamber exit for PR-N. Cold gas operation: S09 (blue solid line, $p_{0}=0.349$ Torr) and $\mathrm{S} 11$ (blue dashed dotted line, $p_{0}=0$ Torr). Plasma operation: S10 (red solid line, $p_{0}=0.349$ Torr) and S12 (red dashed-dotted line, $p_{0}=0$ Torr, extrapolated results).

or Mach 3.6 in PR-N (S12), and $u_{z, \mathrm{ex}}=685.3 \mathrm{~m} \mathrm{~s}^{-1}$ or Mach 2.1 in PR-N (S16). Note that the Mach number is calculated relative to the local sound speed $c_{\mathrm{s}}$, which is dependent on the local temperature of the propellant. The cross sectional area at which $u_{z, \text { ex }}$ reaches $c_{\mathrm{S}}$ is called the sonic surface. ${ }^{39} \mathrm{In}$ PR-C and PR-N, the sonic surface is at the nozzle throat. In a cylindrical geometry like $\mathrm{PR}-\mathrm{O}$, it forms as a parabolic shape slightly upstream of the discharge chamber exit. As the exit velocity is limited by $c_{\mathrm{s}}$, it is beneficial to increase $c_{\mathrm{s}}$ as much as possible by increasing the local temperature of the propellant. The PR-N geometry is the most effective in heating and accelerating the propellant. The peak $u_{z, \text { ex }}$ in S14 is higher than in S16 primarily due to the propellant heating in the nozzle throat, which produces a hotter exhaust plume as well as a higher stagnation pressure $p_{\mathrm{s}}$ in the plenum (see Table I).

Thrust is generated from the linear momentum of the propellant when it leaves the exit surface of the discharge chamber. The thrust force is calculated using the integral form of the general thrust equation

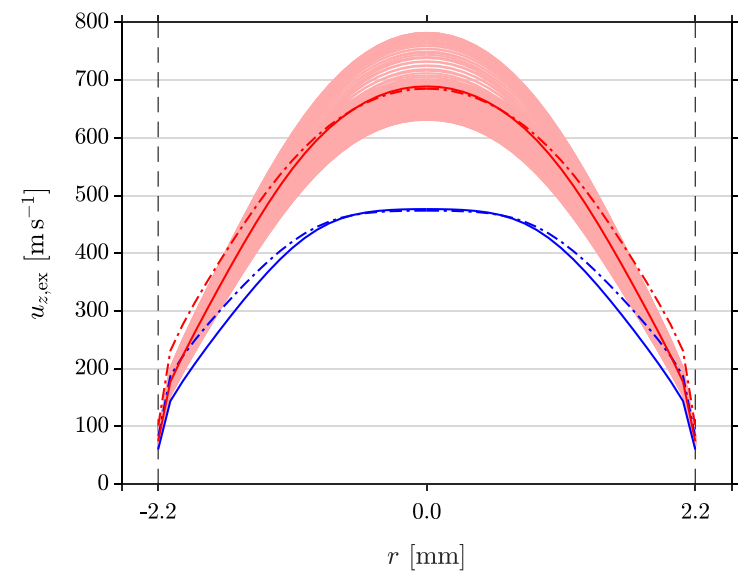

FIG. 17. Cycle average axial velocity $u_{z, \mathrm{ex}}$ across the discharge chamber exit for PR-N. Cold gas operation: S13 (blue solid line, $p_{0}=0.1$ Torr) and S15 (blue dashed dotted line, $p_{0}=0$ Torr). Plasma operation: S14 (red solid line, $p_{0}=0.1$ Torr) and $\mathrm{S} 16$ (red dashed-dotted line, $p_{0}=0$ Torr, extrapolated results). 


$$
F_{\mathrm{t}}=2 \pi \int_{0}^{R} r\left(\rho_{\mathrm{ex}} u_{z, \mathrm{ex}}^{2}+p_{\mathrm{ex}}-p_{0}\right) \mathrm{d} r
$$

with the radial fluid density $\rho_{\text {ex }}$, axial velocity $u_{z, \text { ex }}$, and pressure $p_{\mathrm{ex}}$ profiles across the exit. Figure 18(a) shows a visual representation of Eq. (2). The integration is performed beginning at the central axis at $0 \mathrm{~mm}$ and ending at the cavity wall at $R=2.1 \mathrm{~mm}$ for $\mathrm{PR}-\mathrm{O}$ and $\mathrm{PR}-\mathrm{C}$, and $R=2.2 \mathrm{~mm}$ for $\mathrm{PR}-\mathrm{N}$. As discussed previously, ${ }^{26}$ the integral form is preferred since the radial profiles of the aforementioned parameters are not flat across the exit surface. To ascertain the accuracy of the thrust force calculations, a similar integration of the mass flow rate of the gas $\left(\dot{m}_{\mathrm{ex}}=\rho_{\mathrm{ex}} u_{z, \mathrm{ex}}\right)$ is performed across the exit area and compared to the supplied mass flow rate at the inlet of $\dot{m}=100$ SCCM. The error is found to be in the range of $-5.6 \% \leq \Delta \dot{m}_{\mathrm{ex}} \leq+0.1 \%$, indicating that the results of CFD and CFD-plasma simulations are reasonably precise. Table I lists the nominal thrust force $F_{t}$ at $\dot{m}=100 \mathrm{SCCM}$, with $\Delta \dot{m}_{\mathrm{ex}}$ already taken into account. $F_{t}$ values for simulations run with nonzero $p_{0}$ are greyed out; the primary interest is the performance in a vacuum environment.

The thrust force increases during plasma operation by the following amounts: $+9.5 \%$ in PR-O $+27.6 \%$ in PR-C, $+42.0 \%$ in PR-N (S12), and $+27.2 \%$ in PR $-\mathrm{N}(\mathrm{S} 16)$. The largest increase is seen in S12, due to the heating of the propellant in the nozzle throat. However, it has been established that plasma operation in a vacuum environment drastically changes the discharge characteristics, and the ion density no longer peaks in the nozzle throat, and therefore S12 is not an

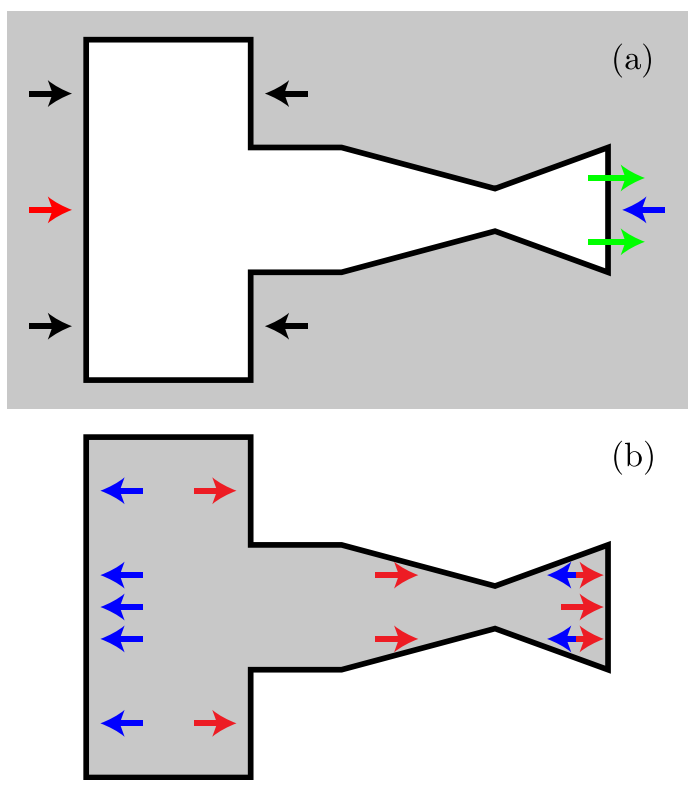

FIG. 18. (a) Force diagram for the general thrust equation. The thrust force is calculated as the external forces acting on the propellant volume and the momentum of the exiting propellant. Arrows represent the terms $\rho_{\mathrm{ex}} u_{z, \mathrm{ex}}^{2}$ (green), $p_{\text {ex }}$ (blue), and $p_{0}$ (red) in Eq. (2). Black arrows also represent $p_{0}$ but may be ignored as they cancel each other out. (b) Force diagram for the internal forces method. Pressure forces within the propellant volume act on the walls of PR and on the background environment at the exit. The thrust force is assumed to be the difference between the positive force vectors (blue) and the negative force vectors (red). The friction force between the boundary layer and the wall is not accounted for. This results in gross overestimation of the calculated thrust force. accurate representation of the performance in reality. Nonetheless, S12 provides very useful information on how to further optimise the geometry of $\mathrm{PR}-\mathrm{N}$ in order to reach a compromise between the desirable features of both plasma confinement and also propellant heating in the nozzle throat. If possible, the optimised geometry can perhaps achieve around $\mathrm{a}+35 \%$ thrust force increase during plasma operation. Again, it must be stressed that these performance metrics and the $F_{\mathrm{t}}$ values represent the instantaneous or pulsed plasma operation performance of $\mathrm{PR}$ at the present power draw, and higher performance is expected when the discharge chamber wall and attains thermal equilibrium with the heated propellant or with higher RF power.

The cycle average specific impulse $I_{\mathrm{sp}}$ calculated using $F_{t}$ is also listed in Table $\mathrm{I}$. The values may be compared with the theoretical maximum specific impulse, given by

$$
\bar{I}_{\mathrm{sp}}=\frac{1}{g} \cdot\left\{\frac{k_{\mathrm{B}} T_{\mathrm{s}}}{m} \cdot \frac{2 \gamma}{\gamma-1} \cdot\left[1-\left(\frac{p_{0}}{p_{\mathrm{s}}}\right)^{\frac{\gamma-1}{\gamma}}\right]\right\}^{\frac{1}{2}},
$$

where $g=9.81 \mathrm{~m} \mathrm{~s}^{-2}$ is the standard gravity, $k_{\mathrm{B}}$ is the Boltzmann constant, $\gamma$ is the adiabatic index, $m$ is the molecular mass of the gas, and $T_{\mathrm{s}}$ and $p_{\mathrm{s}}$ are the stagnation temperature and pressure, respectively. The $p_{0}$ term within the parentheses assumes that the flow at the exit is perfectly expanded such that $p_{\mathrm{ex}}=p_{0}$. For expansion into vacuum, $\bar{I}_{\mathrm{sp}}$ becomes independent of $p_{\mathrm{s}}$ and is solely determined by $T_{\mathrm{s}}$. For $T_{\mathrm{s}}=300 \mathrm{~K}$, the theoretical maximum specific impulse for $\operatorname{Ar}$ gas is $\bar{I}_{\mathrm{sp}}=57.0 \mathrm{~s}$. In S15, the steady state $I_{\mathrm{sp}}=49.1 \mathrm{~s}$ is somewhat lower than $\bar{I}_{\mathrm{sp}}$ due to frictional and viscous losses in the boundary layer, which are unavoidable in practice. In $\mathrm{S} 16$ on the other hand, the transient $I_{\mathrm{sp}}=62.5 \mathrm{~s}$ represents the minimum value achieved immediately after plasma ignition, and the eventual steady state $I_{\mathrm{sp}}$ is expected to be considerably higher after thermal equilibrium is attained (or with higher RF power).

The net pressure force $F_{p}$ on PR can be measured by integrating the total fluid pressure force along all the internal facing walls [illustrated in Fig. 18(b)]. Because ions are treated as being part of the fluid in the CFD-plasma simulations, it is not possible to investigate the plasma pressure forces separately from that of the neutral fluid. The integration is performed radially and excludes the radial component of the pressure force which cancels out on opposite sides of PR anyway. $F_{p}$ obtained by this method is roughly 1.6 to 2.7 times of $F_{\mathrm{t}} \cdot F_{p}$ must not be misinterpreted as the actual thrust force. It is not so, because this method neglects to account for the friction force $F_{\mathrm{bl}}$ between the boundary layer and the wall $^{26}$ and therefore results in a gross overestimation of the actual thrust force. $F_{\mathrm{bl}}$ can be thought of as a force exerted on the propellant by the wall, result in a loss against the momentum that the propellant has gained from the net pressure force imbalance. Alternatively, from the reference frame of the propellant, $F_{\mathrm{bl}}$ acts on the wall in the direction of flow, which is opposite to the intended direction of motion of $\mathrm{PR}$.

While there is no method for experimentally or theoretically measuring $F_{\mathrm{bl}}$, the CFD and CFD-plasma simulations offer a way of obtaining its value by simply calculating the 
difference $F_{\mathrm{t}}-F_{\mathrm{p}}=F_{\mathrm{bl}}$. Since the flow in PR is in the slip regime, boundary layer effects are significant, and it shows in the comparison of the respective values of $F_{\mathrm{bl}}$ and $F_{\mathrm{t}}$ listed in Table I.

\section{F. Power}

Different amounts of RF power are required to drive the $300 \mathrm{~V}$ amplitude sinusoidal RF waveform on the powered electrode in each PR geometry and test case. The cycle average RF power draw $P$ may be extracted by integrating the volumetric and surface energy at each time-step of the RF cycle. Volumetric power deposition $\left(P_{\mathrm{V}}\right)$ in the plasma is dominated by ion-neutral charge exchange collisions but also includes processes such as electron-neutral charge exchange collisions and other volumetric reactions. Surface power deposition $\left(P_{\mathrm{S}}\right)$ is primarily due to ion bombardment in to the self-biased section of the discharge chamber wall shielding the powered electrode, with negligible contribution from other processes like surface recombination and deexcitation reactions. ${ }^{22}$ These values are listed in Table II.

In $\mathrm{S} 02, P=5.01 \mathrm{~W}$, which is in great agreement with the value of $4.8 \mathrm{~W}$ measured with a digital inline voltage/current $(\mathrm{V} / \mathrm{I})$ probe on the $\mathrm{PR}-\mathrm{O}$ experimental setup. While the CFD and CFD-plasma simulation techniques have been fine tuned for PR-O against experimental and theoretical parameters such as pressure, velocity, thrust force, spatiotemporal heating trends, and the self-bias formation, the same techniques applied to PR-C and PR-N and different operating conditions produce results that are reasonable but not guaranteed to be absolutely accurate until they have been tested experimentally. For example, a CFD-plasma simulation of PR-O using a $400 \mathrm{~V}$ amplitude instead of $300 \mathrm{~V}$ shows a RF power draw of $P=16.3 \mathrm{~W}$, which is significantly higher than the experimentally measured value of $10.0 \mathrm{~W}$. This suggests that $P$ is likely to be overestimated at higher RF voltages.

One possible solution is to empirically find the appropriate secondary electron emission coefficient (SEEC) that scales with RF voltages. To explore this issue, simulations S17 and S19 are run exactly the same as S10 and S14, respectively, but with SEEC $=0.05$ instead of 0.1. The simulation results of S17 and S19 have the same general characteristics as S10 and S14, but with a slight scaling down of $n_{\mathrm{i}}$ and $P$ along with the other corresponding fluid and plasma

TABLE II. The total RF power $P$ drawn by the discharge in each CFDplasma simulation, compared with the power deposition in volumetric $\left(P_{\mathrm{V}}\right)$ and surface $\left(P_{\mathrm{S}}\right)$ processes. Also listed is the power efficiency given by the increase in thrust force from cold gas operation to plasma operation, using results extrapolated to $p_{0}=0$ Torr from the listed simulations. All values are averaged over the RF cycle.

\begin{tabular}{lccccccc}
\hline \hline Sim. & Geometry & $\begin{array}{c}P \\
(\mathrm{~W})\end{array}$ & $\begin{array}{c}P_{\mathrm{V}} \\
(\mathrm{W})\end{array}$ & $\begin{array}{c}P_{\mathrm{V}} / P \\
(\%)\end{array}$ & $\begin{array}{c}P_{\mathrm{S}} \\
(\mathrm{W})\end{array}$ & $\begin{array}{r}P_{\mathrm{S}} / P \\
(\%)\end{array}$ & $\begin{array}{c}\Delta F_{\mathrm{t}} / P \\
\left(\mu \mathrm{N} \mathrm{W}^{-1}\right)\end{array}$ \\
\hline S02 & PR-O & 5.01 & 0.34 & 6.87 & 4.67 & 93.1 & $25.8(\mathrm{~S} 04)$ \\
S06 & PR-C & 13.3 & 1.16 & 8.74 & 12.1 & 91.3 & $29.6(\mathrm{~S} 08)$ \\
S10 & PR-N & 12.4 & 2.14 & 17.3 & 10.2 & 82.7 & $48.6(\mathrm{~S} 12)$ \\
S14 & PR-N & 12.0 & 1.97 & 16.4 & 10.0 & 83.6 & $32.4(\mathrm{~S} 16)$ \\
S17 & PR-N & 10.6 & 1.47 & 13.8 & 9.15 & 86.2 & $75.2(\mathrm{~S} 18)$ \\
S19 & PR-N & 8.75 & 1.20 & 13.7 & 7.55 & 86.3 & $24.1(\mathrm{~S} 20)$ \\
\hline \hline
\end{tabular}

parameters (see Tables I and II). It is important to note that $n_{\mathrm{i}}$ and $P$ are also dependent on a number of other factors such as the plasma impedance and the self-bias voltage at the discharge chamber wall. ${ }^{27}$ Each of these factors must be treated rigorously in the CFD-plasma simulations and tested against experiments.

It is evident from Table II that the amount of power $P_{\mathrm{V}}$ deposited volumetrically into the propellant that effects heating is only a small proportion of the total RF power draw $P$. The remainder $P_{\mathrm{S}}$, which is a significant proportion of $P$, is deposited onto the plasma-facing surface of the discharge chamber wall via ion bombardment. This energy is not wasted per se, as ion bombardment is a critical and necessary process for the creation of the secondary electrons that are ultimately responsible for sustaining the gamma mode discharge in PR. Additionally, $P_{\mathrm{S}}$ heats up the discharge chamber wall over time. As $P_{\mathrm{S}}$ is considerably higher than $P_{\mathrm{V}}$, the thrust performance may increase quite significantly when the propellant is in thermal equilibrium with the discharge chamber wall. Unfortunately, this behaviour is not captured on the time scale of the present CFDplasma simulations.

There is an improvement in $\mathrm{P}_{\mathrm{V}} / P$ moving from $\mathrm{PR}-\mathrm{O}$ to the nozzle geometries, with the highest proportion seen in $\mathrm{PR}-\mathrm{N}$. Interestingly, the values of S10 and S14 are very close, as are those of S17 and S19. This is due to the dominant $P_{\mathrm{S}}$, which is directly related to ion dynamics in the plasma sheath along the self-biased section of the discharge chamber wall. Evidence of this is found in the cycle average electric potential on the plasma-facing surface of the discharge chamber wall $\Phi_{\text {wall }}$, which is the main determining factor of ion dynamics in the plasma sheath apart from the SEEC. In Fig. 19, the $\Phi_{\text {wall }}$ profiles of S10 (blue solid line) and S14 (red solid line) track closely to each other along the first half of the discharge chamber wall. The same behaviour is seen with the $\Phi_{\text {wall }}$ profiles of S17 (cyan solid line) and S19 (magenta solid line).

The partitioning of $P_{\mathrm{V}}$ versus $P_{\mathrm{S}}$ is directly related to the discharge impedance. Experimentally, this means that different $P R$ designs require different impedance matching

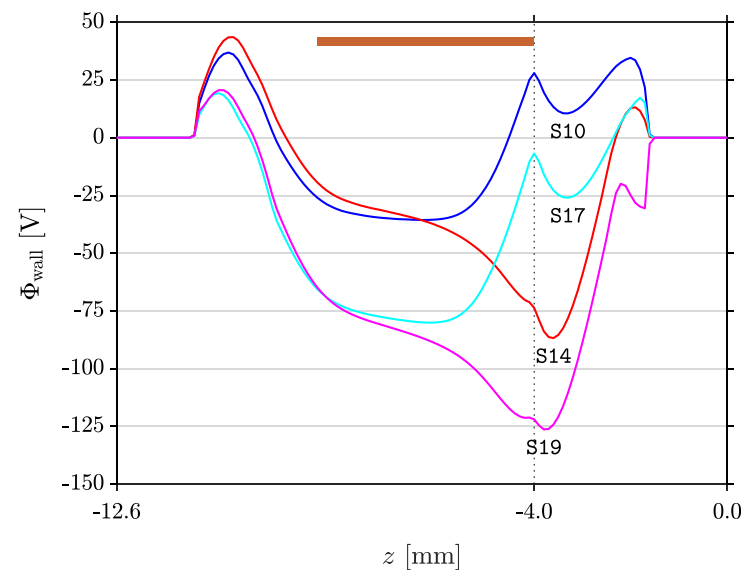

FIG. 19. Cycle average electric potential on the plasma-facing surface of the discharge chamber wall $\Phi_{\text {wall }}$ in S10 (blue solid line), S14 (red solid line), S17 (cyan solid line), and S19 (magenta solid line). Note that $\Phi_{\text {wall }}$ is not necessarily the self-bias voltage. ${ }^{27}$ 
conditions. The CFD-plasma simulations are not affected as the RF voltage amplitude on the powered electrode is a fixed variable, with the assumption of perfect impedance matching in the RF electrical circuit between the powered electrode and the RF power generator. Overall, $P$ tends to scale with $n_{\mathrm{i}}$. A larger number of ions increase the electrical conductivity of the discharge and thus allow for more power to be coupled into the plasma while driving the powered electrode at the same RF voltage. This is analogous to the relation $P=V^{2} / R$.

Power efficiency may be quantified by calculating the increase in thrust force during plasma operation from cold gas operation per unit of supplied RF power. Most values of $\Delta F_{\mathrm{t}} / P$ are the range of $24.1 \mu \mathrm{N} \mathrm{W}^{-1}$ to $32.4 \mu \mathrm{N} \mathrm{W}^{-1}$. For perspective, the current holder of the world record for the highest thrust generated by an electric thruster is the X3 threechannel nested Hall effect thruster. ${ }^{43,44}$ It has a mass of $230 \mathrm{~kg}$ and uses Xe propellant flowing at up to $\dot{m}=2000$ SCCM $=1.95 \times 10^{-4} \mathrm{~kg} \mathrm{~s}^{-1} . \mathrm{X} 3$ produces $5.42 \mathrm{~N}$ of thrust force while drawing $98.4 \mathrm{~kW}$ of power, giving it a power efficiency of $55.1 \mu \mathrm{NW}^{-1}$. Table II shows S10 and S19 both giving surprisingly high power efficiencies. This indicates that propellant heating in the nozzle throat is very desirable, if it is indeed achievable in $p_{0}=0$ Torr conditions. Further optimisation of the nozzle geometry and discharge impedance can potentially allow PR to attain $\Delta F_{\mathrm{t}} / P$ values comparable to $\mathrm{X} 3$ at a fraction of the thruster mass and volume, as well as propellant and power consumption, and be a highly attractive propulsion option on nanosatellite missions.

\section{CONCLUSION}

In summary, this paper investigates the performance of three different geometries of the PR radiofrequency plasma electrothermal microthruster. This is done by means of highly precise CFD-plasma simulations, which offer insights that are otherwise unobtainable through experimentation or theoretical estimation.

The plasma, temperature, velocity, thrust force, and power parameters of PR are discussed in detail, making comparisons between the geometries $\mathrm{PR}-\mathrm{O}, \mathrm{PR}-\mathrm{C}$, and $\mathrm{PR}-\mathrm{N}$, as well as between cold gas and plasma operation modes. During plasma operation, the thrust force is increased by $\sim 30 \%$ with a reasonable power efficiency of $\sim 30 \mu \mathrm{NW}^{-1}$ at a total power draw of $\sim 10 \mathrm{~W}$. These performance metrics reported in this paper represent the instantaneous pulsed plasma operation performance of $\mathrm{PR}$, and higher performance is expected when the discharge chamber wall attains thermal equilibrium with the heated propellant or with higher RF power.

The best performance is found in the sculpted PR-N geometry. A higher ion density is achieved, and more RF power can be coupled into the plasma at a fixed RF voltage. The nozzle geometry not only increases the transit time of the propellant through the discharge chamber, which allows for more effective heating through more numerous ionneutral charge exchange collisions, but is also able to confine the plasma within the discharge chamber due to the formation of a plasma sheath at the nozzle throat. Enhanced recombination in the divergent section of the nozzle ensures that the exhaust plume is essentially neutral, thereby avoiding contamination of solar panels and interference with externally mounted instruments. Future optimisations of PR are recommended to seek a compromise between desirable propellant heating in the nozzle throat and plasma confinement.

${ }^{1}$ E. Gill, P. Sundaramoorthy, J. Bouwmeester, B. Zandbergen, and R. Reinhard, Acta Astronaut. 82, 110 (2013).

${ }^{2}$ J. Virgili and P. C. E. Roberts, Acta Astronaut. 89, 130 (2013).

${ }^{3}$ M. M. Micci and A. D. Ketsdever, Micropropulsion for Small Spacecraft (AIAA, Reston, 2000).

${ }^{4}$ S. Mazouffre, Plasma Sources Sci. Technol. 25, 033002 (2016).

${ }^{5}$ A. M. Baker, A. da Silva Curiel, J. Schaffner, and M. Sweeting, Acta Astronaut. 57, 288 (2005).

${ }^{6}$ A. D. Ketsdever, R. H. Lee, and T. C. Lilly, J. Micromech. Microeng. 15, 2254 (2005).

${ }^{7}$ H. Horisawa and I. Kimura, Vacuum 59, 106 (2000).

${ }^{8}$ H. Horisawa, H. Ashiya, and I. Kimura, in Proceedings of International Electric Propulsion Conference, Toulouse, 2003.

${ }^{9}$ D. A. Lamprou, V. J. Lappas, T. Shimizu, D. Gibbon, and M. Perren, in Proceedings of International Electric Propulsion Conference, Wiesbaden, 2011.

${ }^{10}$ A. N. Grubisic and S. B. Gabriel, J. Propul. Power 32, 810 (2016).

${ }^{11}$ D. M. Goebel and I. Katz, Fundamentals of Electric Propulsion: Ion and Hall Thrusters (Wiley, Hoboken, 2008).

${ }^{12}$ C. Charles and R. W. Boswell, Plasma Sources Sci. Technol. 21, 022002 (2012).

${ }^{13}$ A. Pascale, "Design and construction of a propellant sub-system for SP3's CubeSat plasma thruster," Honours thesis (The Australian National University, Canberra, 2016).

${ }^{14}$ A. Stuchbery, "Development, integration, and testing of the "pocket rocket' plasma thruster," Honours thesis (The Australian National University, Canberra, 2017).

${ }^{15}$ C. Charles, T. S. Ho, A. Pascale, T. Charoy, A. Bennet, A. Stuchbery, R. W. Boswell, W. Liang, L. Raymond, and J. Rivas-Davila, in Proceedings of International Astronautical Congress, Adelaide, 2017.

${ }^{16}$ C. Charles, W. Liang, L. Raymond, J. Rivas-Davila, and R. W. Boswell, Front. Phys. 5, 36 (2017).

${ }^{17}$ C. Charles, R. W. Boswell, and A. Bish, J. Propul. Power 30, 1117 (2014).

${ }^{18}$ A. Greig, C. Charles, R. Hawkins, and R. W. Boswell, Appl. Phys. Lett. 103, 074101 (2013).

${ }^{19}$ A. Greig, C. Charles, N. Paulin, and R. W. Boswell, Appl. Phys. Lett. 105, 054102 (2014).

${ }^{20}$ A. Greig, C. Charles, and R. W. Boswell, Front. Phys. 3, 84 (2015).

${ }^{21}$ A. Greig, C. Charles, and R. W. Boswell, Phys. Plasmas 23, 013508 (2016).

${ }^{22}$ T. S. Ho, C. Charles, and R. W. Boswell, Phys. Plasmas 24, 084501 (2017).

${ }^{23}$ H. Böhrk and M. Auweter-Kurtz, J. Propul. Power 25, 729 (2009).

${ }^{24}$ C. Charles, R. W. Boswell, A. Bish, V. Khayms, and E. F. Scholz, Front. Phys. 4, 19 (2016)

${ }^{25}$ M. A. Kemp and S. D. Kovaleski, IEEE Trans. Plasma Sci. 36, 356 (2008).

${ }^{26}$ T. S. Ho, C. Charles, and R. W. Boswell, Front. Phys. 4, 55 (2017).

${ }^{27}$ T. S. Ho, C. Charles, and R. W. Boswell, "Redefinition of the self-bias voltage in a dielectrically shielded thin sheath RF discharge," J. Appl. Phys. (unpublished).

${ }^{28}$ A. Greig, C. Charles, and R. W. Boswell, Front. Phys. 2, 80 (2015).

${ }^{29}$ A. Greig, C. Charles, and R. W. Boswell, IEEE Trans. Plasma Sci. 42, 2728 (2014).

${ }^{30} \mathrm{~F}$. La Torre, "Gas flow in miniaturized nozzles for micro-thrusters," $\mathrm{Ph} . \mathrm{D}$. thesis (Delft University of Technology, Delft, 2011).

${ }^{31}$ A. Greig, "Pocket Rocket: An electrothermal plasma micro-thruster," Ph.D. thesis (The Australian National University, Canberra, 2015).

${ }^{32}$ N. Mutsukura, K. Kobayashi, and Y. Machi, J. Appl. Phys. 68, 2657 (1990).

${ }^{33}$ M. Surendra and D. B. Graves, Appl. Phys. Lett. 59, 2091 (1991).

${ }^{34}$ M. J. de Graaf, R. P. Dahiya, J. L. Jauberteau, F. J. de Hoog, M. J. F. van de Sande, and D. C. Schram, J. Phys. Colloq. 51, 387 (1990).

${ }^{35}$ R. F. G. Meulenbroeks, D. C. Schram, L. J. M. Jaegers, and M. C. M. van de Sanden, Phys. Rev. Lett. 69, 1379 (1992).

${ }^{36}$ A. Fruchtman, IEEE Trans. Plasma Sci. 39, 530 (2010). 
${ }^{37}$ P. J. Bruggeman, N. Sadeghi, D. C. Schram, and V. Linss, Plasma Sources Sci. Technol. 23, 023001 (2014).

${ }^{38}$ J.-P. Booth, D. Marinov, M. Foucher, O. Guaitella, D. Bresteau, L. Cabaret, and C. Drag, J. Instrum. 10, C11003 (2015).

${ }^{39}$ H. R. Murphy and D. R. Miller, J. Phys. Chem. 88, 4474 (1984).

${ }^{40}$ A. Fruchtman, Plasma Sources Sci. Technol. 17, 024016 (2008).

${ }^{41}$ A. Fruchtman, J. Phys. D: Appl. Phys. 50, 473002 (2017).
${ }^{42}$ M. N. Kogan, Rarefied Gas Dynamics (Springer, New York, 1969).

${ }^{43}$ S. J. Hall, R. E. Florenz, A. D. Gallimore, H. Kamhawi, D. L. Brown, J. E. Polk, D. M. Goebel, and R. R. Hofer, in Proceedings of Joint Propulsion Conference, American Institute of Aeronautics and Astronautics, Reston, Virginia, 2014.

${ }^{44}$ S. J. Hall, S. E. Cusson, and A. D. Gallimore, in Proceedings of International Electric Propulsion Conference, Kobe, 2015. 